\title{
On the Exact Values of HZ-Index for the Graphs under Operations
}

\author{
Dalal Awadh Alrowaili $\mathbb{D},{ }^{1}$ Saira Javed $\mathbb{D}{ }^{2}$, and Muhammad Javaid $\mathbb{D}^{2}$ \\ ${ }^{1}$ Department of Mathematics, College of Science, Jouf University, Sakaka 2014, Saudi Arabia \\ ${ }^{2}$ Department of Mathematics, School of Science, University of Management and Technology, Lahore, Pakistan
}

Correspondence should be addressed to Muhammad Javaid; javaidmath@gmail.com

Received 4 August 2021; Accepted 25 October 2021; Published 20 November 2021

Academic Editor: Ali Ahmad

Copyright ( 2021 Dalal Awadh Alrowaili et al. This is an open access article distributed under the Creative Commons Attribution License, which permits unrestricted use, distribution, and reproduction in any medium, provided the original work is properly cited.

Topological index (TI) is a function from the set of graphs to the set of real numbers that associates a unique real number to each graph, and two graphs necessarily have the same value of the TI if these are structurally isomorphic. In this note, we compute the $\mathrm{HZ}$ - index of the four generalized sum graphs in the form of the various Zagreb indices of their factor graphs. These graphs are obtained by the strong product of the graphs $G$ and $D_{k}(G)$, where $D_{k} \in\left\{S_{k}, R_{k}, Q_{k}, T_{k}\right\}$ represents the four generalized subdivision-related operations for the integral value of $k \geq 1$ and $D_{k}(G)$ is a graph that is obtained by applying $D_{k}$ on $G$. At the end, as an illustration, we compute the $\mathrm{HZ}$ - index of the generalized sum graphs for exactly $k=1$ and compare the obtained results.

\section{Introduction}

A structural formula of a chemical compound is represented by a molecular graph, where atoms and bonds between atoms are represented by the vertices and edges of the molecular graphs, respectively. A topological index (TI) is a mathematical tool which associates a real number to a graph under certain conditions. For two graphs, a TI remains constant if the graphs are isomorphic (see [1-3]). These are used to study different physical attributes, biological activities, and chemical reactivities such as viscosity, critical temperatures (boiling, freezing, melting, and flash points) $[4,5]$, vapor pressure, surface tension, stability, weight, density, solubility, and connectivity [6-8] in the field of chemical engineering, pharmaceutical industries, and drugs discoveries. TIs are also used in the subject of cheminformatics to study the quantitative structural activity and property relationships (see [9-11]).

In 1947, the very first TI is introduced by Winer to check the critical temperature of paraffin [12]. Trinajstic and Gutman (1972) [13] defined the first and second Zagreb indices that are used to compute the different structure base characteristics of the molecular graphs. After that, many degree, distance, and polynomials based TIs came into existence but the degree-based indices got more attention of the researchers (see [14-16]). For various results on TIs of different graphs, see [17-20]. In 2008, Zhou and Trinajstić defined the general sum connectivity (GSC) index and discussed its various properties [21]. Shirdel et al. [22] studied the concept of hyper-Zagreb index ( $\mathrm{HZ}$ - index) as a particular case of the GSC index. In addition, the results for the index HZ under the operation of Cartesian, composition, join, and disjunction of graphs can be found in [23-25].

On the other hand, for the studies of the complex graphs, operations for graphs play a key role. Yan et al. (2007) defined four types of operations related to the subdivision of $G$ and computed the Wiener indices of the derived graphs $D_{1}(G)$, where $D_{1} \in\left\{S_{1}, R_{1}, Q_{1}, T_{1}\right\}$ [26]. Taeri et al. (2009) gave the construction of the $D_{1}$-sum graphs $G_{D_{1}}+H$ (Cartesian product of $F_{1}(G)$ and $H$ ) and computed their Wiener indices, where $H$ and $G$ are assumed to be two connected graphs [27]. Furthermore, Deng et al. [28], Akhter and Imran [29], Chu et al. [30], and Liu et al. [31] computed the various indices of these graphs with the help of the Cartesian product.

Liu et al. (2019) [32] extended these operations for any integral value of $k$ and obtained the generalized derived graphs $D_{k}(G)$ of the graph $G$, where $D_{k} \in\left\{S_{k}, R_{k}, Q_{k}, T_{k}\right\}$. Moreover, using the concept of Cartesian product of graphs, they constructed the generalized sum graphs or $D_{k}$-sum graphs (denoted by $G_{D_{k}}+H$ ) and computed their first and second Zagreb indices. 
Javaid et al. (2021) [33] redefined these graphs using strong product and computed their Zagreb indices (first and second). In this development, we compute hyper-Zagreb indices ( $\mathrm{HZ}$ - index) for these graphs in terms of various degree-based TIs of their factor graphs, where these generalized sum graphs are obtained with the help of strong product. The remaining paper is settled as follows. Section 2 contains the notations and key concepts which are utilized in methodology, Section 3 deals main results, and Section 4 covers examples and conclusion.

\section{Preliminaries}

This section explains the basic definitions and terminologies.

Definition 1. Let $G=(V(G), E(G))$ be a (molecular) graph with $V(G)$ and $E(G)$ as sets of vertices and edges, respectively. The degree of a vertex $v \in V(G)$ is the number of edges which are incident on $v$ and denoted by $d(v)$.

Definition 2 (see $[13,34]$ ). For a graph $G$, the first, second, and forgotten Zagreb indices are defined as follows: $M_{1}(G)=$ $\sum_{z \in V(G)} d^{2}(z)=\sum_{z t \in E(G)}[d(z)+d(t)], \quad M_{2}(G)=\sum_{z t \in E(G)}$ $[d(z) \times d(t)], \quad$ and $\quad F(G)=\sum_{z \in V(G)} d^{3}(z)=\sum_{z t \in E(G)}$ $\left[d^{2}(z)+d^{2}(t)\right]$

These indices have been used to find the various properties of molecular graphs such as entropy, $\pi$-electron energy, and heat capacity. These are also used in the studies of the molecular structural relationships such as QSPR and QSAR [13, 35-37]. However, the hyper-Zagreb index of a graph $(G)$ (given below) is studied by Shirdel et al. in 2013 [22]:

$$
\mathrm{HZ}(G)=\sum_{y z \in E(G)}[d(y)+d(z)]^{2} .
$$

Definition 3 (see [32]). For some integral value of $k \geq 1$, the graphs obtained by the generalized subdivision-related operations are defined as follows:

(i) $S_{k}(G)$ is a graph that is obtained by inserting $k$ vertices in each edge of $G$

(ii) $R_{k}(G)$ is a graph obtained from $S_{k}(G)$ by joining the vertices which are adjacent in $G$

(iii) $Q_{k}(G)$ is a graph obtained from $S_{k}(G)$ by joining the new vertices which are on the incident edges in $G$ for each of its vertex

(iv) $T_{k}(G)$ is obtained from $S_{k}(G)$ after using both $R_{k}$ and $Q_{k}$, respectively

For $k=3$, see Figure 1 .

Definition 4 (see [33]). Let $G_{1}$ and $G_{2}$ be two graphs, $D_{k} \in\left\{S_{k}, R_{k}, Q_{k}, T_{k}\right\}$ be generalized subdivision-related operations, and $D_{k}\left(G_{1}\right)$ be a graph obtained using $D_{k}$ on $G_{1}$ having edge-set $E\left(D_{k}\left(G_{1}\right)\right)$ and vertex-set $V\left(D_{k}\left(G_{1}\right)\right)$. The generalized sum graph $G_{1} \otimes_{D_{k}} G_{2}$ under the operation of strong product is a graph having vertex-set $V\left(G_{1} \otimes_{D_{k}} G_{2}\right)=$ $V\left(D_{k}\left(G_{1}\right)\right) \times V\left(G_{2}\right)=\left(V\left(G_{1}\right) \cup k\left(E\left(G_{1}\right)\right)\right) \times V\left(G_{2}\right)$ such that two vertices $\left(r_{1}, s_{1}\right)$ and $\left(r_{2}, s_{2}\right)$ of $V\left(G_{1_{D}} \otimes G_{2}\right)$ are adjacent iff $\left[r_{1}=r_{2}\right.$ in $V\left(G_{1}\right)$ and $s_{1}$ is adjacent to $s_{2}$ in $\left.E\left(G_{2}\right)\right]$ or $\left[s_{1}=s_{2}\right.$ in $V\left(G_{2}\right)$ and $s_{1}$ is adjacent to $s_{2}$ in $\left.E\left(G_{1}\right)\right]$ or $\left[r_{1}\right.$ is adjacent to $r_{2}$ in $E\left(D_{k}\left(G_{1}\right)\right)$ and $s_{1}$ is adjacent to $s_{2}$ in $E\left(G_{2}\right)$ ], where $k \geq 1$ is a positive integer. For more explanation, see Figures 2 and 3 .

\section{Main Results}

The main developments are covered by this section.

Theorem 1. For $k \geq 1$, the HZ-index of $G_{1} \otimes_{S_{k}} G_{2}$ is

$$
\begin{aligned}
& \mathrm{HZ}\left(G_{1} \otimes_{S_{k}} G_{2}\right)=8 e_{G_{1}} M_{1}\left(G_{2}\right)+n_{G_{1}} \mathrm{HZ}\left(G_{2}\right)+4 e_{G_{2}} M_{1}\left(G_{1}\right)+4 e_{G_{1}} \mathrm{HZ}\left(G_{2}\right)+M_{1}\left(G_{1}\right) \mathrm{HZ}\left(G_{2}\right)+4 M_{1}\left(G_{1}\right) M_{1}\left(G_{2}\right) \\
& +n_{G_{2}} \mathrm{HZS}_{1}\left(G_{1}\right)+4 e_{G_{2}} M_{1} S_{1}\left(G_{1}\right)+2 e_{G_{1}} M_{1}\left(G_{2}\right)+4 e_{G_{2}} \mathrm{HZ} \mathrm{S}_{1}\left(G_{2}\right)+2 M_{1}\left(G_{2}\right) M_{1} S_{1}\left(G_{1}\right) \\
& +M_{1}\left(G_{2}\right) \mathrm{HZS}_{1}\left(G_{1}\right)+16(k-1) e_{G_{1}}\left[n_{G_{2}}+M_{1}\left(G_{2}\right)+4 e_{G_{2}}\right]+\mathrm{HZ}_{1} G_{1} F\left(G_{2}\right)+2 M_{1}\left(G_{2}\right) \operatorname{HZS}_{1}\left(G_{1}\right) \\
& +2 M_{1}\left(G_{2}\right) M_{1}\left(S\left(G_{1}\right)\right)+2 F\left(G_{2}\right) M_{1}\left(S\left(G_{1}\right)\right)+16 e_{G_{1}} e_{G_{2}}+4 e_{G_{1}} F\left(G_{2}\right)+2 e_{G_{2}} M_{1}\left(G_{1}\right) \\
& +4(k-1) e_{G_{1}}\left[8 e_{G_{2}}+2 \mathrm{HZ}\left(G_{2}\right)+8 M_{1}\left(G_{2}\right)\right] \text {. }
\end{aligned}
$$

Proof. Let the degree of a vertex $(r, s) \in G_{1} \otimes_{S_{k}} G_{2}$ be denoted by $d(r, s)$ :

$$
\begin{aligned}
\mathrm{HZ}\left(G_{1} \rrbracket_{S_{k}} G_{2}\right)= & \sum_{\left(r_{1}, s_{1}\right)\left(r_{2}, s_{2}\right) \in E\left(G_{1} \otimes_{s_{k}} G_{2}\right)}\left[d\left(r_{1}, s_{1}\right)+d\left(r_{2}, s_{2}\right)\right]^{2} \\
& =\sum_{r \in V\left(G_{1}\right)} \sum_{s_{1} s_{2} \in E\left(G_{2}\right)}\left[d\left(r, s_{1}\right)+d\left(r, s_{2}\right)\right]^{2}+\sum_{r_{1} r_{2} \in E\left(s_{k}\left(G_{1}\right)\right)} \sum_{s e V\left(G_{2}\right)}\left[d\left(r_{1}, s\right)+d\left(r_{2}, s\right)\right]^{2} \\
& +\sum_{r_{1} r_{2} \in E\left(s_{k}\left(G_{1}\right)\right)} \sum_{s_{1} s_{2} \in V\left(G_{2}\right)}\left[d\left(r_{1}, s_{1}\right)+d\left(r_{2}, s_{2}\right)\right]^{2}=\sum_{A}+\sum_{B}+\sum_{C} .
\end{aligned}
$$




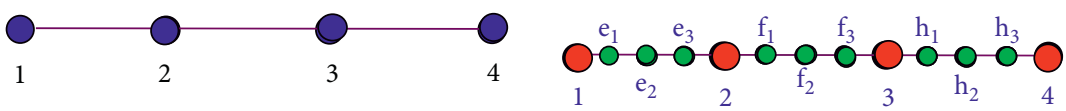

(a)

(b)

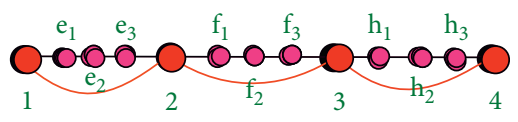

(c)

\section{$\mathrm{f}_{2}$}

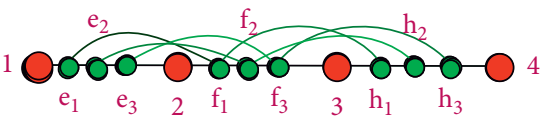

(d)

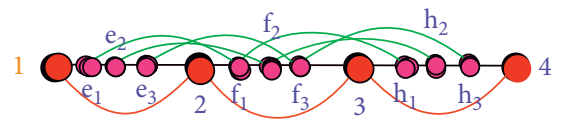

(e)

Figure 1: (a) $G_{1} \cong P_{4}$, (b) $S_{3}\left(P_{4}\right)$, (c) $R_{3}\left(P_{4}\right),\left(\right.$ d) $Q_{3}\left(P_{4}\right)$ and (e) $T_{3}\left(P_{4}\right)$.

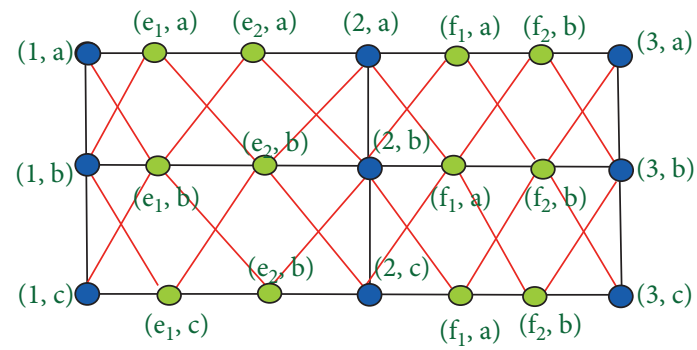

(a)

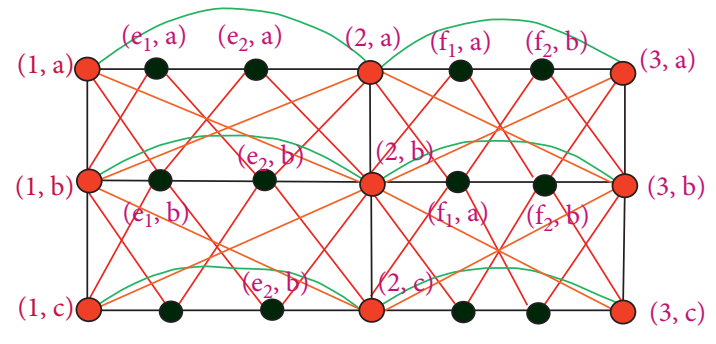

(b)

Figure 2: (a) $P_{3} \rrbracket_{S_{2}} P_{3}$ and (b) $P_{3} \rrbracket_{R_{2}} P_{3}$.

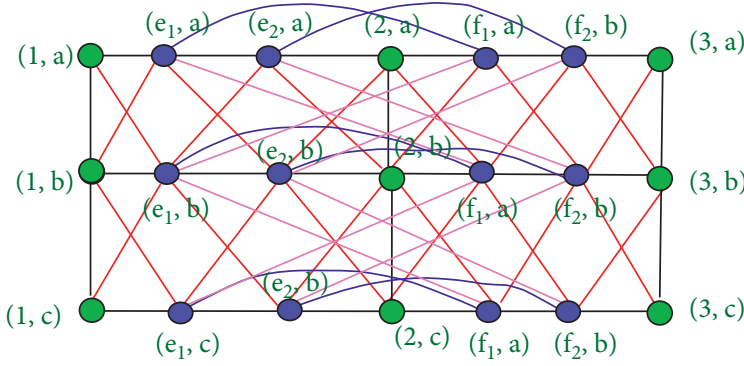

(a)

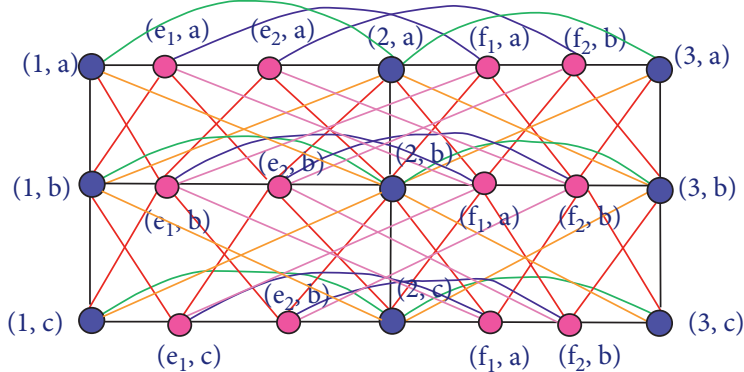

(b)

Figure 3: (a) $P_{3} \otimes_{Q_{2}} P_{3}$ and (b) $P_{3} \otimes_{T_{2}} P_{3}$. 


\section{Consider}

$$
\begin{aligned}
& \sum_{A}=\sum_{r \in V\left(G_{1}\right)} \sum_{s_{1} s_{2} \in E\left(G_{2}\right)}\left[d\left(r, s_{1}\right)+d\left(r, s_{2}\right)\right]^{2} \\
& =\sum_{r \in V\left(G_{1}\right)} \sum_{s_{1} s_{2} \in E\left(G_{2}\right)}\left[2 d(r)+d\left(s_{1}\right)+d\left(s_{2}\right)+d(r)\left(d\left(s_{1}\right)+d\left(s_{2}\right)\right)\right]^{2} \\
& =\sum_{r \in V\left(G_{1}\right)} \sum_{s_{1} s_{2} \in E\left(G_{2}\right)}\left[4 d(r)\left(d\left(s_{1}\right)+d\left(s_{2}\right)\right)+\left(d^{2}\left(s_{1}\right)+d\left(s_{2}\right)+2 d\left(s_{1}\right) d\left(s_{2}\right)\right)+4 d^{2}(r)+2 d(r)\left(d^{2}\left(s_{1}\right)+d^{2}\left(s_{2}\right)\right.\right. \\
& \left.\left.+2 d\left(s_{1}\right) d\left(s_{2}\right)\right)+d^{2}(r)\left(d^{2}\left(s_{1}\right)+d^{2}\left(s_{2}\right)+2 d\left(s_{1}\right) d\left(s_{2}\right)\right)+4 d^{2}(r)\left(d\left(s_{1}\right)+d\left(s_{2}\right)\right)\right] \\
& =8 e_{G_{1}} M_{1}\left(G_{2}\right)+n_{G_{1}} \mathrm{HZ}\left(G_{2}\right)+4 e_{G_{2}} M_{1}\left(G_{1}\right)+4 e_{G_{1}} \mathrm{HZ}\left(G_{2}\right)+M_{1}\left(G_{1}\right) \mathrm{HZ}\left(G_{2}\right)+4 M_{1}\left(G_{1}\right) M_{1}\left(G_{2}\right) \text {, } \\
& \sum_{B}=\sum_{r_{1} r_{2} \in E\left(S_{k}\left(G_{1}\right)\right)} \sum_{s \in V\left(G_{2}\right)}\left[d\left(r_{1}, s\right)+d\left(r_{2}, s\right)\right]^{2}=\sum_{\substack{r_{1} \in V\left(G_{1}\right), r_{2} \in V\left(S_{k}\left(G_{1}\right)-G_{1}\right)}} \sum_{s \in V\left(G_{2}\right)}\left[d\left(r_{1}, s\right)+d\left(r_{2}, s\right)\right]^{2} \\
& +\sum_{\substack{r_{1}, r_{2} \epsilon \\
V\left(S_{k}\left(G_{1}\right)-G_{1}\right)}} \sum_{s \in V\left(G_{2}\right)}\left[d\left(r_{1}, s\right)+d\left(r_{2}, s\right)\right]^{2}=\sum_{B_{1}}+\sum_{B_{2}} \\
& \sum_{B_{1}}=\sum_{\substack{r_{1} r_{2} \in E\left(S_{k}\left(G_{1}\right)\right) \\
r_{1} \in V\left(G_{1}\right), r_{2} \in V\left(S_{k}\left(G_{1}\right)\right)-V\left(G_{1}\right)}} \sum_{s \in V\left(G_{2}\right)}\left[d\left(r_{1}, s\right)+d\left(r_{2}, s\right)\right]^{2} \\
& =\sum_{\substack{r_{1} r_{2} \in E\left(S_{k}\left(G_{1}\right)\right) \\
r_{1} \in V\left(G_{1}\right), r_{2} \in V\left(S_{k}\left(G_{1}\right)\right)-V\left(G_{1}\right)}} \sum_{s \in V\left(G_{2}\right)}\left[\left(d\left(r_{1}\right)+d\left(r_{2}\right)\right)+d(s)+\left(d\left(r_{1}\right)+d\left(r_{2}\right)\right) d(s)\right]^{2} \\
& =\sum_{\substack{r_{1} r_{2} \in E\left(S_{k}\left(G_{1}\right)\right) \\
r_{1} \in V\left(G_{1}\right), r_{2} \in V\left(S_{k}\left(G_{1}\right)\right)-V\left(G_{1}\right)}} \sum_{s \in V\left(G_{2}\right)}\left[\left(d^{2}\left(r_{1}\right)+d^{2}\left(r_{2}\right)+2 d\left(r_{1}\right) d\left(r_{2}\right)\right)+2 d(s)\left(d\left(r_{1}\right)+d\left(r_{2}\right)\right)+d^{2}(s)\right. \\
& +2 d(s)\left(d^{2}\left(r_{1}\right)+d^{2}\left(r_{2}\right)+2 d\left(r_{1}\right) d\left(r_{2}\right)\right)+2 d^{2}(s)\left(d\left(r_{1}\right)+d\left(r_{2}\right)\right)+d^{2}(s)\left(d^{2}\left(r_{1}\right)+d^{2}\left(r_{2}\right)\right) \\
& =n_{G_{2}} \mathrm{HZS}_{1}\left(G_{1}\right)+4 e_{G_{2}} M_{1} S_{1}\left(G_{1}\right)+2 e_{G_{1}} M_{1}\left(G_{2}\right)+4 e_{G_{2}} \mathrm{HZS}_{1}\left(G_{1}\right)+2 M_{1}\left(G_{2}\right) M_{1} S_{1}\left(G_{1}\right) \\
& +M_{1}\left(G_{2}\right) \mathrm{HZS}_{1}\left(G_{1}\right) \\
& \sum_{B_{2}}=\sum_{\substack{r_{1} r_{2} \in E\left(S_{k}\left(G_{1}\right)\right) \\
r_{1}, s_{2} \in V\left(S_{k}\left(G_{1}\right)\right)-V\left(G_{1}\right)}} \sum_{s \in V\left(G_{2}\right)}\left[d\left(r_{1}, s\right)+d\left(r_{2}, s\right)\right]^{2} \\
& =\sum_{\substack{r_{1} r_{2} \in E\left(S_{k}\left(G_{1}\right)\right) \\
r_{1}, r_{2} \in V\left(S_{k}\left(G_{1}\right)\right)-V\left(G_{1}\right)}} \sum_{s \in V\left(G_{2}\right)}\left[d\left(r_{1}\right)+d\left(r_{1}\right) d(s)+d\left(r_{2}\right)+d\left(r_{2}\right) d(s)\right]^{2} \\
& =\sum_{\substack{r_{1} r_{2} \in E\left(S_{k}\left(G_{1}\right)\right) \\
r_{1}, r_{2} \in V\left(S_{k}\left(G_{1}\right)\right)-V\left(G_{1}\right)}} \sum_{s \in V\left(G_{2}\right)}[4+4 d(s)]^{2}=\sum_{\substack{r_{1} r_{2} \in E\left(S_{k}\left(G_{1}\right)\right) \\
r_{1}, r_{2} \in V\left(S_{k}\left(G_{1}\right)\right)-V\left(G_{1}\right)}} \sum_{s \in V\left(G_{2}\right)}\left[16+16 d^{2}(s)+32 d(s)\right] .
\end{aligned}
$$


Since in this case $\left|E\left(S_{k}\left(G_{1}\right)\right)\right|=(k-1)\left|E\left(G_{1}\right)\right|$, we have

$$
\begin{aligned}
& =\sum_{s \in V\left(G_{2}\right)} 16(k-1) e_{G_{1}}\left[1+d^{2}(s)+2 d(s)\right] \\
& \sum_{C}=\sum_{r_{1} r_{2} \in E\left(s_{k}\left(G_{1}\right)\right)}\left[d\left(r_{1}, s_{1}\right)+d\left(r_{2}, s_{2}\right)\right]^{2}=\sum_{\substack{r_{1} r_{2} \in E\left(S_{k}\left(G_{1}\right)\right) \\
r_{1} \in V\left(G_{1}\right), r_{2} \in\left(\left(S_{k}\left(G_{1}\right)\right)-V\left(G_{1}\right)\right.}} \sum_{s_{1} s_{2} \in V\left(G_{2}\right)}\left[d\left(r_{1}, s_{1}\right)+d\left(r_{2}, s_{2}\right)\right]^{2} \\
& +\sum_{\substack{r_{1} r_{2} \in E\left(S_{k}\left(G_{1}\right)\right) \\
r_{1}, r_{2} \in V\left(\left(S_{k}\left(G_{1}\right)\right)-V\left(G_{1}\right)\right.}} \sum_{\substack{s_{1} s_{2} \in V\left(G_{2}\right)\\
}}\left[d\left(r_{1}, s_{1}\right)+d\left(r_{2}, s_{2}\right)\right]^{2}=\sum_{C_{1}}+\sum_{C_{2}} \\
& \sum_{C_{1}}=\sum_{r_{1} r_{2} \in E\left(S_{k}\left(G_{1}\right)\right)} \sum_{s_{1} s_{2} \in V\left(G_{2}\right)}\left[d\left(r_{1}, z_{1}\right)+d\left(r_{2}, s_{2}\right)\right]^{2} \\
& r_{1} \in V\left(G_{1}\right), r_{2} \epsilon\left(\left(S_{k}\left(G_{1}\right)\right)-V\left(G_{1}\right)\right. \\
& =\sum_{\substack{r_{1} \in V\left(G_{1}\right), r_{2} \in V\left(\left(S_{k}\left(G_{1}\right)\right)\right)-V\left(G_{1}\right)}} \sum_{s_{1} s_{2} \in V\left(G_{2}\right)}\left[\left(d\left(r_{1}\right)+d\left(r_{2}\right)\right)+d\left(s_{1}\right)+d\left(r_{1}\right) d\left(s_{1}\right)+d\left(r_{2}\right) d\left(s_{2}\right)\right]^{2} \\
& =\mathrm{HZG}_{1} F\left(G_{2}\right)+2 M_{1}\left(G_{2}\right) \mathrm{HZS}_{1}\left(G_{1}\right)+2 M_{1}\left(G_{2}\right) M_{1}\left(S\left(G_{1}\right)\right) \\
& +2 F\left(G_{2}\right) M_{1}\left(S\left(G_{1}\right)\right)+16 e_{G_{1}} e_{G_{2}}+4 e_{G_{1}} F\left(G_{2}\right)+2 e_{G_{2}} M_{1}\left(G_{1}\right) \text {, } \\
& \sum_{C_{2}}=\sum_{\substack{r_{1} r_{2} \in E\left(S_{k}\left(G_{1}\right)\right) \\
r_{1}, r_{2} \in V\left(\left(S_{k}\left(G_{1}\right)\right)\right)-V\left(G_{1}\right)}} \sum_{\substack{s_{1} s_{2} \in V\left(G_{2}\right)\\
}}\left[d\left(r_{1}, s_{1}\right)+d\left(r_{2}, s_{2}\right)\right]^{2} \\
& =\sum_{\substack{r_{1} r_{2} \in E\left(S_{k}\left(G_{1}\right)\right) \\
r_{1}, r_{2} E V\left(\left(s_{k}\left(G_{1}\right)\right)\right)-V\left(G_{1}\right)}} \sum_{s_{1} s_{2} \in V\left(G_{2}\right)}\left[4+2\left(d\left(s_{1}\right)+d\left(s_{2}\right)\right)\right]^{2} \\
& =\sum_{\substack{r_{1} r_{2} \in E\left(s_{k}\left(G_{1}\right)\right) \\
r_{1}, r_{2} \in V\left(\left(S_{k}\left(G_{1}\right)\right)\right)-V\left(G_{1}\right)}} \sum_{\substack{s_{1} s_{2} \in V\left(G_{2}\right)\\
}}\left[16+4\left(d\left(z_{1}\right)+d\left(z_{2}\right)\right)^{2}+16\left(d\left(z_{1}\right)+d\left(z_{2}\right)\right)\right] \\
& =4(k-1) e_{G_{1}}\left[8 e_{G_{2}}+2 \mathrm{HZ}\left(G_{2}\right)+8 M_{1}\left(G_{2}\right)\right] \text {. }
\end{aligned}
$$

Hence, we obtained our required result.

Theorem 2. For $k \geq 1$, the HZ-index of $G_{1} \unrhd_{R_{k}} G_{2}$ is

$$
\begin{aligned}
\mathrm{HZ}\left(G_{1} \bigotimes_{R_{k}} G_{2}\right)= & 8\left[n_{G_{2}}+6 e_{G_{2}}\right] F\left(G_{1}\right)+\left[n_{G_{1}}+20 e_{G_{1}}\right] F\left(G_{2}\right)+8 F\left(G_{1}\right) F\left(G_{2}\right)+24 e_{G_{2}} M_{1}\left(G_{1}\right)+36 e_{G_{1}} M_{1}\left(G_{2}\right) \\
& +24 M_{1}\left(G_{1}\right) M_{1}\left(G_{2}\right)+24 F\left(G_{1}\right) M_{1}\left(G_{2}\right)+8 n_{G_{2}} e_{G_{1}}+8(k-1) e_{G_{1}}\left[n_{G_{2}}+F\left(G_{2}\right)+4 e_{G_{2}}+3 M_{1}\left(G_{2}\right)\right] \\
& +48 e_{G_{1}} e_{G_{2}}+12 F\left(G_{2}\right) M_{1}\left(G_{1}\right)+2\left[M_{2}\left(G_{2}\right)\left[4 e_{G_{1}}+n_{G_{1}}\right]+k\left[n_{G_{2}}+6 e_{G_{2}}+3 M_{1}\left(G_{2}\right)+2 M_{2}\left(G_{2}\right)\right]\right. \\
& +\left[\frac{1}{2} \sum_{v \in V\left(G_{1}\right)}\left(d_{G_{1}}^{4}(v)-d_{G_{1}}^{3}(v)\right)+\sum_{v \in V\left(G_{1}\right)} r d_{G_{1}}(u) d_{G_{1}}(v)+\sum_{v \in V\left(G_{1}\right)} d_{G_{1}}^{2}(v) \sum_{u \in V\left(G_{1}\right)} d_{G_{1}}(u)-2 M_{2}\left(G_{1}\right)\right] \\
& \left.+M_{1}\left(G_{1}\right)\left[5 e_{2}+5 M_{1}\left(G_{2}\right)+5 M_{2}\left(G_{2}\right)\right]+k\left[M_{3}\left(G_{1}\right)+2 M_{2}\left(G_{1}\right)\right]\left[6 e_{G_{2}}+3 M_{1}\left(G_{2}\right)+2 M_{2}\left(G_{2}\right)+n_{G_{2}}\right]\right] \\
& +2 e_{G_{1}} M_{1}\left(G_{2}\right) .
\end{aligned}
$$


Proof. Let the degree of a vertex $(r, s) \in G_{1} \otimes_{R_{k}} G_{2}$ be denoted by $d(r, s)$ :

$$
\begin{aligned}
& \operatorname{HZ}\left(G_{1} \otimes_{R_{k}} G_{2}\right)=\sum_{\left(r_{1}, s_{1}\right)\left(r_{2}, s_{2}\right) \in E\left(G_{1} \otimes_{R_{k}} G_{2}\right)}\left[d\left(r_{1}, s_{1}\right)+d\left(r_{2}, s_{2}\right)\right]^{2} \\
& \cdot \sum_{r \in V\left(G_{1}\right)} \sum_{s_{1} s_{2} \in E\left(G_{2}\right)}\left[d\left(r, s_{1}\right)+d\left(r, s_{2}\right)\right]^{2}+\sum_{s \in V\left(G_{2}\right)} \sum_{r_{1} r_{2} \in E\left(R_{k}\left(G_{1}\right)\right)}\left[d\left(r_{1}, s\right)+d\left(r_{2}, s\right)\right]^{2} \\
& +\sum_{r_{1} r_{2} \in E\left(R_{k}\left(G_{1}\right)\right)} \sum_{s_{1} s_{2} \in V\left(G_{2}\right)}\left[d\left(r_{1}, s_{1}\right)+d\left(r_{2}, s_{2}\right)\right]^{2}=\sum_{A}+\sum_{B}+\sum_{C} \\
& \sum_{A}=\sum_{r \in V\left(G_{1}\right)} \sum_{s_{1} s_{2} \in E\left(G_{2}\right)}\left[d\left(r, s_{1}\right)+d\left(r, s_{2}\right)\right]^{2} \\
& =\sum_{r \in V\left(G_{1}\right)} \sum_{s_{1} s_{2} \in E\left(G_{2}\right)}\left[2 d(r)+d\left(s_{1}\right)+2 d(r) d\left(s_{1}\right)+2 d(r)+d\left(s_{2}\right)+2 d(r) d\left(s_{2}\right)\right]^{2} \\
& =\sum_{r \in V\left(G_{1}\right)} \sum_{s_{1} s_{2} \in E\left(G_{2}\right)}\left[4 d(r)+d\left(s_{1}\right)+d\left(s_{2}\right)+2 d(r)\left(d\left(s_{1}\right)+d\left(s_{2}\right)\right)\right]^{2} \\
& =\sum_{r \in V\left(G_{1}\right)} \sum_{s_{1} s_{2} \in E\left(G_{2}\right)}\left[\left(4 d^{2}(r)+1+4 d(r)\right)\left(d^{2}\left(s_{1}\right)+d^{2}\left(s_{2}\right)+2 d\left(s_{1}\right) d\left(s_{2}\right)\right)+\left(8 d(r)+16 d^{2}(r)\right)\right. \\
& \left.\times\left(d\left(s_{1}\right)+d\left(s_{2}\right)\right)+16 d^{2}(r)\right] \\
& =\operatorname{HZG}_{2}\left[4 M_{1}\left(G_{1}\right)+n_{G_{1}}+8 e_{G_{1}}\right]+16 M_{1}\left(G_{2}\right)\left[e_{G_{1}}+M_{1}\left(G_{1}\right)\right]+16 M_{1}\left(G_{1}\right) e_{G_{2}} \\
& \text { OR } \\
& =8 e_{G_{2}} M_{1}\left(G_{1}\right)+n_{G_{1}} F\left(G_{2}\right)+4 M_{1}\left(G_{1}\right) F\left(G_{2}\right)+8 e_{G_{1}} M_{1}\left(G_{2}\right)+8 M_{1}\left(G_{1}\right) M_{1}\left(G_{2}\right)+8 e_{G_{1}} F\left(G_{2}\right) \\
& +2\left[4 M_{1}\left(G_{1}\right)\left[e_{G_{2}}+M_{1}\left(G_{2}\right)+M_{2}\left(G_{2}\right)\right]+4 e_{G_{1}}\left[M_{1}\left(G_{2}\right)+2 M_{2}\left(G_{2}\right)\right]+M_{2}\left(G_{2}\right) n_{G_{1}}\right], \\
& \sum_{B}=\sum_{r_{1} r_{2} \in E\left(R_{k}\left(G_{1}\right)\right)} \sum_{s \in V\left(G_{2}\right)}\left[d\left(r_{1}, s\right)+d\left(r_{2}, s\right)\right]^{2}=\sum_{\substack{r_{1} r_{2} \in E\left(R_{k}\left(G_{1}\right)\right) \\
r_{1}, r_{2} \in V\left(G_{1}\right)}} \sum_{s \in V\left(G_{2}\right)}\left[d\left(r_{1}, s\right)+d\left(r_{2}, s\right)\right]^{2} \\
& +\sum_{\substack{r_{1} r_{2} \in E\left(R_{k}\left(G_{1}\right)\right) r_{1} \in V\left(G_{1}\right), r_{2} \in V\left(R_{k}\left(G_{1}\right)\right)-V\left(G_{1}\right)}}\left[d\left(r_{1}, s\right)+d\left(r_{2}, s\right)\right]^{2}+\sum_{\substack{r_{1} r_{2} \in E\left(R_{k}\left(G_{1}\right)\right) \\
r_{1}, r_{2} \in V\left(R_{k}\left(G_{1}\right)\right)-V\left(G_{1}\right)}} \sum_{s \in V\left(G_{2}\right)}\left[d\left(r_{1}, s\right)+d\left(r_{2}, s\right)\right]^{2} \\
& =\sum_{B_{1}}+\sum_{B_{2}}+\sum_{B_{3}} \\
& \sum_{B_{1}}=\sum_{\substack{r_{1} r_{2} \in E\left(R_{k}\left(G_{1}\right)\right) \\
r_{1}, r_{2} \in V\left(G_{1}\right)}} \sum_{s \in V\left(G_{2}\right)}\left[d\left(r_{1}, s\right)+d\left(r_{2}, s\right)\right]^{2} \\
& =\sum_{\substack{r_{1} r_{2} \in E\left(R_{k}\left(G_{1}\right)\right) \\
r_{1}, r_{2} \in V\left(G_{1}\right)}} \sum_{s \in V\left(G_{2}\right)}\left[d\left(r_{1}\right)+d(s)+d\left(r_{1}\right) d(s)+d\left(r_{2}\right)+d(s)+d\left(r_{2}\right) d(s)\right]^{2} \\
& =\sum_{\substack{r_{1} r_{2} \in E\left(R_{k}\left(G_{1}\right)\right) \\
r_{1}, r_{2} \in V\left(G_{1}\right)}} \sum_{s \in V\left(G_{2}\right)}\left[d\left(r_{1}\right)+d\left(r_{2}\right)+2 d(s)+d(s)\left(d\left(r_{1}\right)+d\left(r_{2}\right)\right)\right]^{2} \\
& =\sum_{\substack{r_{1} r_{2} \in E\left(R_{k}\left(G_{1}\right)\right) \\
r_{1}, r_{2} \in V\left(G_{1}\right)}} \sum_{s \in V\left(G_{2}\right)}\left[4 d^{2}\left(r_{1}\right)+4 d^{2}(s)+4 d\left(4 d^{2}\left(r_{2}\right)+8 d\left(r_{1}\right) d(s)+8 d\left(r_{1}\right) d\left(r_{2}\right)+8 d(s) d\left(r_{2}\right)\right.\right. \\
& \left.+4 d^{2}(s)\left(d^{2}\left(r_{1}\right)+d^{2}\left(r_{2}\right)+2 d(s) d^{2}\left(r_{1}\right)\right)+8 d\left(s_{1}\right)\left(d^{2}\left(r_{1}\right)+d^{2}\left(r_{2}\right)+2 d(s) d^{2}\left(r_{1}\right)\right)+8 d^{2}\left(s_{1}\right)\left(d\left(r_{1}\right)+d\left(r_{2}\right)\right)\right] \\
& =4 e_{G_{1}} M_{1}\left(G_{2}\right)+\left[4 n_{G_{2}}+4 M_{1}\left(G_{2}\right)+16 e_{G_{2}}\right] \mathrm{HZ}\left(G_{1}\right)+16 e_{G_{2}} M_{1}\left(G_{1}\right)+8 M_{1}\left(G_{1}\right) M_{1}\left(G_{2}\right) \text {. } \\
& =4 n_{G_{2}} F\left(G_{1}\right)+2 e_{G_{1}} M_{1}\left(G_{2}\right)+4 M_{1}\left(G_{2}\right) F\left(G_{1}\right)+8 e_{G_{2}} M_{1}\left(G_{1}\right)+4 M_{1}\left(G_{1}\right) M_{1}\left(G_{2}\right)+16 e_{G_{2}} F\left(G_{1}\right) \\
& +2\left[4 M_{2}\left(G_{1}\right)\left[n_{G_{2}}+4 e_{G_{2}}+M_{1}\left(G_{2}\right)\right]+2 M_{1}\left(G_{1}\right)\left[M_{1}\left(G_{2}\right)+2 e_{G_{2}}\right]+e_{G_{1}} M_{1}\left(G_{2}\right)\right] \text {, }
\end{aligned}
$$




$$
\begin{aligned}
& \sum_{B_{2}}=\sum_{\substack{r_{1} r_{2} \in E\left(R_{k}\left(G_{1}\right)\right) \\
r_{1} \in V\left(G_{1}\right), r_{2} \in V\left(R_{k}\left(G_{1}\right)\right)-V\left(G_{1}\right)}} \sum_{s \in V\left(G_{2}\right)}\left[d\left(r_{1}, s\right)+d\left(r_{2}, s\right)\right]^{2} \\
& =\sum_{\substack{r_{1} r_{2} \in E\left(R_{k}\left(G_{1}\right)\right) \\
r_{1} \in V\left(G_{1}\right), r_{2} \in V\left(R_{k}\left(G_{1}\right)\right)-V\left(G_{1}\right)}} \sum_{s \in V\left(G_{2}\right)}\left[d\left(r_{1}\right)+d\left(r_{2}\right)+d(s)+\left(d\left(r_{1}\right)+d\left(r_{2}\right)\right) d(s)\right]^{2} \\
& =\sum_{\substack{r_{1} r_{2} \in E\left(R_{k}\left(G_{1}\right)\right) \\
r_{1} \in V\left(G_{1}\right), r_{2} \in V\left(R_{k}\left(G_{1}\right)\right)-V\left(G_{1}\right)}} \sum_{s \in V\left(G_{2}\right)}\left[2 d\left(r_{1}\right)+3 d(s)+2 d\left(r_{1}\right) d(s)+2\right]^{2} \\
& =4 n_{G_{2}} F\left(G_{1}\right)+18 e_{G_{1}} M_{1}\left(G_{2}\right)+8 e_{G_{1}} n_{G_{2}}+40 e_{G_{2}} M_{1}\left(G_{1}\right)+48 e_{G_{1}} e_{G_{2}}+8 n_{G_{2}} M_{1}\left(G_{1}\right) \\
& +4 F\left(G_{1}\right) M_{1}\left(G_{2}\right)+12 M_{1}\left(G_{1}\right) M_{1}\left(G_{2}\right)+16 e_{G_{2}} F\left(G_{1}\right) \\
& \sum_{B_{3}}=\sum_{\substack{r_{1} r_{2} \in E\left(R_{k}\left(G_{1}\right)\right) \\
r_{1}, r_{2} \in V\left(R_{k}\left(G_{1}\right)\right)-V\left(G_{1}\right)}} \sum_{s \in V\left(G_{2}\right)}\left[d\left(r_{1}, s\right)+d\left(r_{2}, s\right)\right]^{2} \\
& =\sum_{\substack{r_{1} r_{2} \in E\left(R_{k}\left(G_{1}\right)\right) \\
r_{1}, r_{2} \in V\left(R_{k}\left(G_{1}\right)\right)-V\left(G_{1}\right)}} \sum_{s \in V\left(G_{2}\right)}\left[d\left(r_{1}\right)+d\left(r_{1}\right) d(s)+d\left(r_{2}\right)+d\left(r_{2}\right) d(s)\right]^{2} \\
& =\sum_{\substack{r_{1} r_{2} \in E\left(R_{k}\left(G_{1}\right)\right) \\
r_{1}, r_{2} \in V\left(R_{k}\left(G_{1}\right)\right)-V\left(G_{1}\right)}} \sum_{s \in V\left(G_{2}\right)}[4+4 d(s)]^{2}=\sum_{\substack{r_{1} r_{2} \in E\left(R_{k}\left(G_{1}\right)\right) \\
r_{1}, r_{2} \in V\left(R_{k}\left(G_{1}\right)\right)-V\left(G_{1}\right)}} \sum_{s \in V\left(G_{2}\right)}\left[16+16 d^{2}(s)+32 d(s)\right] \\
& =\sum_{s \in V\left(G_{2}\right)} 16(k-1) e_{G_{1}}\left[1+d^{2}(s)+2 d(s)\right]=16(k-1) e_{G_{1}}\left[n_{G_{2}}+M_{1}\left(G_{2}\right)+4 e_{G_{2}}\right] \\
& \sum_{C}=\sum_{r_{1} r_{2} \in E\left(R_{k}\left(G_{1}\right)\right)} \sum_{s_{1} s_{2} \in V\left(G_{2}\right)}\left[d\left(r_{1}, s_{1}\right)+d\left(r_{2}, s_{2}\right)\right]^{2}=\sum_{\substack{r_{1} r_{2} \in E\left(R_{k}\left(G_{1}\right)\right) \\
r_{1}, r_{2} \in V\left(G_{1}\right)}} \sum_{s_{1} s_{2} \in V\left(G_{2}\right)}\left[d\left(r_{1}, s_{1}\right)+d\left(r_{2}, s_{2}\right)\right]^{2} \\
& +\sum_{\substack{r_{1} r_{2} \in E\left(R_{k}\left(G_{1}\right)\right) \\
r_{1} \in V\left(G_{1}\right), r_{2} \in V\left(R_{k}\left(G_{1}\right)\right)-V\left(G_{1}\right)}} \sum_{s_{1} s_{2} \in V\left(G_{2}\right)}\left[d\left(r_{1}, s_{1}\right)+d\left(r_{2}, s_{2}\right)\right]^{2} \\
& +\sum_{\substack{r_{1} r_{2} \in E\left(R_{k}\left(G_{1}\right)\right) \\
r_{1}, r_{2} \in V\left(R_{k}\left(G_{1}\right)\right)-V\left(G_{1}\right)}} \sum_{s_{1} s_{2} \in V\left(G_{2}\right)}\left[d\left(r_{1}, s_{1}\right)+d\left(r_{2}, s_{2}\right)\right]^{2}=\sum_{C_{1}}+\sum_{C_{2}}+\sum_{C_{3}} \\
& \sum_{C_{1}}=\sum_{\substack{r_{1} r_{2} \in E\left(R_{k}\left(G_{1}\right)\right) \\
r_{1}, r_{2} \in V\left(G_{1}\right)}} \sum_{s_{1} s_{2} \in V\left(G_{2}\right)}\left[d\left(r_{1}, s_{1}\right)+d\left(r_{2}, s_{2}\right)\right]^{2} \\
& =\sum_{\substack{r_{1} r_{2} \in E\left(R_{k}\left(G_{1}\right)\right) \\
r_{1}, r_{2} \in V\left(G_{1}\right)}} \sum_{s_{1} s_{2} \in V\left(G_{2}\right)}\left[d\left(r_{1}\right)+d\left(s_{1}\right)+d\left(r_{1}\right) d\left(s_{1}\right)+d\left(r_{2}\right)+d\left(s_{2}\right)+d\left(r_{2}\right) d\left(s_{2}\right)\right]^{2} \\
& =\sum_{\substack{\left.r_{1} r_{2} \in E\left(R_{k}\left(G_{1}\right)\right)\right) \\
r_{1}, r_{2} \in V\left(G_{1}\right)}} \sum_{\substack{s_{1} s_{2} \in V\left(G_{2}\right) \\
+2}}\left[\left(d\left(r_{1}\right)+d\left(s_{1}\right)+d\left(r_{1}\right) d\left(s_{1}\right)\right)^{2}+\left(d\left(r_{1}\right)+d\left(s_{2}\right)+d\left(r_{2}\right) d\left(s_{2}\right)\right)^{2}\right] \\
& +2\left[4 d\left(r_{1}\right) d\left(r_{2}\right)+2\left[d\left(r_{1}\right) d\left(s_{2}\right)+d\left(r_{2}\right) d\left(s_{1}\right)\right]+d\left(s_{1}\right) d\left(s_{2}\right)\right. \\
& \left.+4 d\left(r_{1}\right) d\left(r_{2}\right) d\left(s_{1}\right) d\left(s_{2}\right)+4 d\left(r_{1}\right) d\left(r_{2}\right)\left[d\left(s_{1}\right)+d\left(s_{2}\right)\right]+2\left[d\left(r_{1}\right)+d\left(r_{2}\right)\right] d\left(s_{1}\right) d\left(s_{2}\right)\right] \\
& =8 e_{G_{2}} F\left(G_{1}\right)+2 e_{G_{1}} F\left(G_{2}\right)+4 F\left(G_{1}\right) F\left(G_{2}\right)+4 M_{1}\left(G_{1}\right) M_{1}\left(G_{2}\right)+4 M_{1}\left(G_{1}\right) F\left(G_{2}\right)+8 M_{1}\left(G_{2}\right) F\left(G_{1}\right) \\
& +2\left[8 M_{2}\left(G_{1}\right) e_{G_{2}}+2 M_{1}\left(G_{1}\right) M_{1}\left(G_{2}\right)+2 M_{2}\left(G_{2}\right) e_{G_{1}}+8 M_{2}\left(G_{1}\right)\left[M_{2}\left(G_{2}\right)+M_{1}\left(G_{2}\right)\right]+4 M_{1}\left(G_{1}\right) M_{2}\left(G_{2}\right)\right] \text {, }
\end{aligned}
$$




$$
\begin{aligned}
& \sum_{C_{2}}=\sum_{\substack{r_{1} r_{2} \in E\left(R_{k}\left(G_{1}\right)\right) \\
r_{1} \in V\left(G_{1}\right), r_{2} \in V\left(\left(R_{k}\left(G_{1}\right)\right)-V\left(G_{1}\right)\right.}} \sum_{\substack{s_{1} s_{2} \in V\left(G_{2}\right)\\
}}\left[d\left(r_{1}, s_{1}\right)+d\left(r_{2}, s_{2}\right)\right]^{2} \\
& =\sum_{\substack{r_{1} r_{2} \in E\left(R_{k}\left(G_{1}\right)\right) \\
r_{1} \in V\left(G_{1}\right), r_{2} \in V\left(\left(R_{k}\left(G_{1}\right)\right)-V\left(G_{1}\right)\right.}} \sum_{\substack{s_{1} s_{2} \in V\left(G_{2}\right) \\
r_{2}}}\left[d\left(r_{1}\right)+d\left(s_{1}\right)+d\left(r_{1}\right) d\left(s_{1}\right)+d\left(r_{2}\right)+d\left(r_{2}\right) d\left(s_{2}\right)\right]^{2} \\
& =\quad \sum_{r_{1} r_{2} \in E\left(R_{k}\left(G_{1}\right)\right)} \sum_{s_{1} s_{2} \in V\left(G_{2}\right)}\left[\left(d\left(r_{1}\right)+d\left(s_{1}\right)+d\left(r_{1}\right) d\left(s_{1}\right)\right)^{2}+\left(d\left(r_{2}\right)+d\left(r_{2}\right) d\left(s_{2}\right)\right)^{2}\right] \\
& r_{1} \in V\left(G_{1}\right), r_{2} \in V\left(\left(R_{k}\left(G_{1}\right)\right)-V\left(G_{1}\right)\right. \\
& +2\left[\left(d\left(r_{1}\right)+d\left(s_{1}\right)+d\left(r_{1}\right) d\left(s_{1}\right)\right)\left(d\left(r_{2}\right)+d\left(r_{2}\right) d\left(s_{2}\right)\right)\right] \\
& =8 e_{G_{2}} F\left(G_{1}\right)+32 e_{G_{1}} e_{G_{2}}+4 F\left(G_{1}\right) F\left(G_{2}\right)+4 M_{1}\left(G_{1}\right) M_{1}\left(G_{2}\right)+8 F\left(G_{1}\right) M_{1}\left(G_{2}\right) \\
& +2 e_{G_{1}} F\left(G_{2}\right)+4 M_{1}\left(G_{1}\right) F\left(G_{2}\right)+8 e_{G_{1}} F\left(G_{2}\right)+16 e_{G_{1}} M_{1}\left(G_{2}\right)+8 e_{G_{1}}\left[M_{1}\left(G_{2}\right)+M_{2}\left(G_{2}\right)\right] \\
& +2\left[M_{2}\left(R_{1}\left(G_{1}\right)\right)-4 M_{2}\left(G_{1}\right)\right]\left[2 e_{G_{2}}+2 M_{1}\left(G_{2}\right)+2 M_{2}\left(G_{2}\right)\right], \\
& \sum_{C_{3}}=\sum_{\substack{r_{1} r_{2} \in E\left(\left(R_{k}\left(G_{1}\right)\right) \\
r_{1}, r_{2} \in V\left(\left(R_{k}\left(G_{1}\right)\right)-V\left(G_{1}\right)\right.\right.}} \sum_{\substack{s_{1} s_{2} \in V\left(G_{2}\right)\\
}}\left[d\left(r_{1}, s_{1}\right)+d\left(r_{2}, s_{2}\right)\right]^{2} \\
& =\sum_{\substack{r_{1} r_{2} \in\left(\left(R_{k}\left(G_{1}\right)\right)\right) \\
r_{1}, r_{2} \in V\left(\left(R_{k}\left(G_{1}\right)\right)-V\left(G_{1}\right)\right.}} \sum_{\substack{s_{1} s_{2} \in V\left(G_{2}\right)\\
}}\left[d\left(r_{1}\right)+d\left(r_{2}\right)+d\left(r_{1}\right) d\left(s_{1}\right)+d\left(r_{2}\right) d\left(s_{2}\right)\right]^{2} \\
& =\left[\left(2+2 d\left(s_{1}\right)\right)^{2}+\left(2+2 d\left(s_{2}\right)\right)^{2}+2\left[2+2 d\left(s_{1}\right)\right]\left(2+2 d\left(s_{2}\right)\right)\right) \\
& =8(k-1) e_{G_{1}}\left[2 e_{G_{2}}+F\left(G_{2}\right)+2 M_{1}\left(G_{2}\right)\right]+16(k-1) e_{G_{1}}\left[e_{G_{2}}+M_{1}\left(G_{2}\right)+M_{2}\left(G_{2}\right)\right] .
\end{aligned}
$$

Hence, we reached at our required result.

Theorem 3. For $k \geq 1$, the HZ-index of $G_{1} \otimes_{Q_{k}} G_{2}$ is

$$
\begin{aligned}
& \mathrm{HZ}\left(G_{1} \otimes_{R_{k}} G_{2}\right)=2(k-1)\left[F\left(G_{1}\right)+2 M_{2}\left(G_{1}\right)\right]\left[3 n_{G_{2}}+5 M_{1}\left(G_{1}\right)+14 e_{G_{2}}+F\left(G_{2}\right)\right] \\
& +k\left[n_{G_{2}}+6 e_{G_{2}}+3 M_{1}\left(G_{2}\right)+F\left(G_{2}\right)\right] \\
& {\left[M_{4}\left(G_{1}\right)-2 F\left(G_{1}\right)+2 M_{2}\left(G_{1}\right)-4 M_{2}\left(G_{1}\right)+\sum_{u \in V\left(G_{1}\right)} d^{2}(u) \sum_{v \in N(u)} d(v)\right]+6 e_{G_{2}} M_{1}\left(G_{1}\right)} \\
& +10 e_{G_{2}} F\left(G_{2}\right)+3 F\left(G_{1}\right) F\left(G_{2}\right)+6 M_{1}\left(G_{1}\right) M_{1}\left(G_{2}\right)+F\left(G_{2}\right)\left[n_{G_{1}}+3 M_{1}\left(G_{1}\right)+6 e_{G_{2}}+4 M_{2}\left(G_{1}\right)\right] \\
& +F\left(G_{1}\right)\left[n_{G_{2}}+7 M_{1}\left(G_{2}\right)\right]+6 e_{G_{2}} M_{2}\left(G_{1}\right)+8 M_{2}\left(G_{1}\right)\left[e_{G_{2}}+M_{1}\left(G_{2}\right)\right]+2\left[k \left[n_{G_{2}}+6 e_{G_{2}}+3 M_{1}\left(G_{2}\right)\right.\right. \\
& \left.+2 M_{2}\left(G_{2}\right)\right]\left[\frac{1}{2} \sum_{u \in V\left(G_{1}\right)} d_{G_{1}}^{4}(v)-d_{G_{1}}^{3}(v)+\sum_{u \in V\left(G_{1}\right)} t d_{G_{1}}(u) d_{G_{1}}(v)+\sum_{u \in V\left(G_{1}\right)} d_{G_{1}}^{2}(v) \sum_{\substack{u \in V\left(G_{1}\right) \\
u v \in E\left(G_{1}\right)}} d_{G_{1}}(u)\right. \\
& \left.-2 M_{2}\left(G_{1}\right)\right]+M_{2}\left(G_{2}\right)\left[4 e_{G_{1}}+n_{G_{1}}\right]+2 e_{G_{1}} M_{1}\left(G_{2}\right)+M_{1}\left(G_{1}\right)\left[5 e_{2}+5 M_{1}\left(G_{2}\right)+5 M_{2}\left(G_{2}\right)\right] \\
& \left.+k\left[M_{3}\left(G_{1}\right)+2 M_{2}\left(G_{1}\right)\right]\left[6 e_{G_{2}}+3 M_{1}\left(G_{2}\right)+2 M_{2}\left(G_{2}\right)+n_{G_{2}}\right]\right] .
\end{aligned}
$$


Proof. Let the degree of a vertex $(r, s) \in G_{1} \otimes_{Q_{k}} G_{2}$ be denoted by $d(r, s)$ :

$$
\begin{aligned}
& \operatorname{HZ}\left(G_{1} \otimes_{Q_{k}} G_{2}\right)=\sum_{\left(r_{1}, s_{1}\right)} \sum_{\left(r_{2}, s_{2}\right) \in E\left(G_{1} \otimes_{s_{k}} G_{2}\right)}\left[d\left(r_{1}, s_{1}\right)+d\left(r_{2}, s_{2}\right)\right]^{2} \\
& =\sum_{r \in V\left(G_{1}\right)} \sum_{s_{1} s_{2} \in E\left(G_{2}\right)}\left[d\left(r, s_{1}\right)+d\left(r, s_{2}\right)\right]^{2}+\sum_{s \in V\left(G_{2}\right)} \sum_{r_{1} r_{2} \in E\left(Q_{k}\left(G_{1}\right)\right)}\left[d\left(r_{1}, s\right)+d\left(r_{2}, s\right)\right]^{2} \\
& +\sum_{r_{1} r_{2} \in E} \sum_{\left(Q_{k}\left(G_{1}\right)\right)}\left[d\left(r_{1}, s_{1}\right)+d\left(r_{2}, s_{2}\right)\right]^{2}=\sum_{A}+\sum_{B}+\sum_{C} \\
& \sum_{A}=\sum_{r \in V\left(G_{1}\right)} \sum_{s_{1} s_{2} \in E\left(G_{2}\right)}\left[d\left(r, s_{1}\right)+d\left(r, s_{2}\right)\right]^{2} \\
& =8 e_{1} M_{1}\left(G_{2}\right)+n_{1} \mathrm{HZ}\left(G_{2}\right)+4 e_{2} M_{1}\left(G_{1}\right)+4 e_{1} \mathrm{HZ}\left(G_{2}\right)+M_{1}\left(G_{1}\right) \mathrm{HZ}\left(G_{2}\right)+4 M_{1}\left(G_{1}\right) M_{1}\left(G_{2}\right) \\
& \text { OR } \\
& =2\left|E\left(H_{2}\right)\right| M_{1}\left(H_{1}\right)+\left|V\left(H_{1}\right)\right| F\left(H_{2}\right)+M_{1}\left(H_{1}\right) F\left(H_{2}\right) \\
& +4\left|E\left(H_{1}\right)\right| M_{1}\left(H_{2}\right)+2 M_{1}\left(H_{1}\right) M_{1}\left(H_{2}\right)+4\left|E\left(H_{1}\right)\right| F\left(H_{2}\right) \\
& +2\left[M_{1}\left(G_{1}\right) e_{G_{2}}+M_{1}\left(G_{1}\right)\left[M_{1}\left(G_{2}\right)+M_{2}\left(G_{2}\right)\right]+2 e_{G_{1}}\left[M_{1}\left(G_{2}\right)+2 M_{2}\left(G_{2}\right)\right]+M_{2}\left(G_{2}\right) n_{G_{1}}\right] \\
& \sum_{B}=\sum_{s \in V\left(G_{2}\right)} \sum_{r_{1} r_{2} \in E\left(Q_{k}\left(G_{1}\right)\right)}\left[d\left(r_{1}, s\right)+d\left(r_{2}, s\right)\right]^{2} \\
& +\sum_{s \in V\left(G_{2}\right)} \sum_{\substack{r_{1} r_{2} \in E\left(Q_{k}\left(G_{1}\right)\right) \\
r_{1}, r_{2} \in V\left(Q_{k}\left(G_{1}\right)\right)-V\left(G_{1}\right)}}\left[d\left(r_{1}, s\right)+d\left(r_{2}, s\right)\right]^{2} \\
& \sum_{B_{1}}=\sum_{s \in V\left(G_{2}\right)} \sum_{\substack{r_{1} r_{2} \in E\left(Q_{k}\left(G_{1}\right)\right) \\
r_{1} \in V\left(G_{1}\right), r_{2} \in V\left(Q_{k}\left(G_{1}\right)\right)-V\left(G_{1}\right)}}\left[d\left(r_{1}, s\right)+d\left(r_{2}, s\right)\right]^{2} \\
& =\sum_{s \in V\left(G_{2}\right)} \sum_{\substack{r_{1} r_{2} \in E\left(Q_{k}\left(G_{1}\right)\right) \\
r_{1} \in V\left(G_{1}\right), r_{2} \in V\left(Q_{k}\left(G_{1}\right)\right)-V\left(G_{1}\right)}}\left[d\left(r_{1}\right)+d(s)+d\left(r_{1}\right) d(r)+d\left(r_{2}\right)+d\left(r_{2}\right) d(s)\right]^{2} \\
& =\sum_{\substack{\left.r_{1} r_{2} \in E\left(Q_{k}\left(G_{1}\right)\right) \\
s_{1} \in V\left(H_{1}\right)\right) s_{2} \in V\left(Q_{k}\left(H_{1}\right)-H_{1}\right)}} \sum_{s \in V\left(G_{2}\right)}\left[\left(d\left(r_{1}\right)+d(s)+d\left(r_{1}\right) d(s)\right)^{2}\left(d\left(r_{2}\right)+d\left(r_{2}\right) d(s)\right)^{2}\right. \\
& \left.+2\left[d\left(r_{1}\right)+d(s)+d\left(r_{1}\right) d(s)\right]\left[d\left(r_{2}\right)+d(s)+d\left(r_{2}\right) d(s)\right]\right] .
\end{aligned}
$$

Consider $r_{1} \in V\left(G_{1}\right)$ and $d^{2}\left(r_{1}\right)$ occurs $d\left(r_{1}\right)$ times. Thus,

$$
D_{1}=\sum_{\substack{r_{1} r_{2} \in E\left(Q\left(G_{1}\right)\right), r_{1} \in V\left(G_{1}\right), r_{2} \in V\left(Q\left(G_{1}\right)\right)-V\left(G_{1}\right)}} d^{3}\left(r_{1}\right)=F\left(G_{1}\right) .
$$

Let

$$
D_{2}=\sum_{\substack{r_{1} r_{2} \in E\left(Q\left(G_{1}\right)\right), r_{1} \in V\left(G_{1}\right), r_{2} \in V\left(Q\left(G_{1}\right)\right)-V\left(G_{1}\right)}} d^{2}\left(s_{2}\right),
$$


as $s_{2}=u v \epsilon E\left(G_{1}\right)$ and $d^{2}\left(s_{2}\right)$ occurs two times. Therefore,

$$
\begin{aligned}
D_{2}= & 2 \sum_{s_{2}=u v e V\left(Q\left(G_{1}\right)\right)-V\left(G_{1}\right)}[d(u)+d(v)]^{2}=2 \sum_{u v e E\left(G_{1}\right)}\left[d^{2}(u)+d^{2}(v)+2 d(u) d(v)\right]=2\left[F\left(G_{1}\right)+2 M_{2}\left(G_{1}\right)\right], \\
\sum_{B_{1}}= & n_{G_{2}} F\left(G_{1}\right)+2 e_{G_{1}} M_{1}\left(G_{2}\right)+M_{1}\left(G_{2}\right) F\left(G_{1}\right)+4 e_{G_{2}}\left[M_{1}\left(G_{1}\right)+F\left(G_{1}\right)\right]+2 M_{1}\left(G_{1}\right) M_{1}\left(G_{2}\right) \\
& +2 n_{G_{2}}\left[F\left(G_{1}\right)+2 M_{2}\left(G_{1}\right)\right]+2 M_{1}\left(G_{2}\right)\left[F\left(G_{1}\right)+2 M_{2}\left(G_{1}\right)\right]+8 e_{G_{2}}\left[F\left(G_{1}\right)+2 M_{2}\left(G_{1}\right)\right] \\
& +2\left[\left[M_{3}\left(G_{1}\right)+2 M_{2}\left(G_{1}\right)\right]\left[n_{G_{2}}+4 e_{G_{2}}+M_{1}\left(G_{2}\right)\right]+2 M_{1}\left(G_{1}\right)\left[2 e_{G_{2}}+M_{1}\left(G_{2}\right)\right]\right] \\
\sum_{B_{2}}= & \sum_{\substack{r_{1} r_{2} \in E\left(Q_{k}\left(G_{1}\right)\right) \\
r_{1} r_{2} \in V\left(Q_{k}\left(G_{1}\right)\right)-V\left(G_{1}\right)}} \sum_{s \in V\left(G_{2}\right)}\left[d\left(r_{1}, s\right)+d\left(r_{2}, s\right)\right]^{2} .
\end{aligned}
$$

Now, assume $\Sigma_{B_{2}}=\Sigma_{B_{3}}+\Sigma_{B_{4}}$ as follows:

$$
\begin{aligned}
\sum_{B_{3}}= & \sum_{\substack{r_{1} r_{2} \in E\left(Q_{k}\left(G_{1}\right)\right) \\
r_{1} r_{2} \in V\left(Q_{k}\left(G_{1}\right)\right)-V\left(G_{1}\right)}} \sum_{s e V\left(G_{2}\right)}\left[\left(d\left(r_{1}\right)+d\left(r_{1}\right) d(s)\right)^{2}+\left(d\left(r_{2}\right)+d\left(r_{2}\right) d(s)\right)^{2}\right. \\
& \left.+2\left[\left(d\left(r_{1}\right)+d\left(r_{1}\right) d(s)\right)\left(d\left(r_{2}\right)+d\left(r_{2}\right) d(s)\right)\right]\right] \\
= & 2(k-1)\left[\left(F\left(G_{1}\right)+2 M_{2}\left(G_{1}\right)\right)\left(n_{G_{2}}+M_{1}\left(G_{2}\right)+4 e_{G_{2}}\right)+\left(M_{3}\left(G_{1}\right)+2 M_{2}\left(G_{1}\right)\right)\left(n_{G_{2}}+4 e_{G_{2}}+M_{1}\left(G_{2}\right)\right)\right], \\
\sum_{B_{4}}= & \sum_{\substack{r_{1} r_{2} \in E\left(Q_{k}\left(G_{1}\right)\right) \\
r_{1} r_{2} \in V\left(Q_{k}\left(G_{1}\right)\right)-V\left(G_{1}\right)}} \sum_{s \in V\left(G_{2}\right)}\left[d\left(r, s_{1}\right)+d\left(r, s_{2}\right)\right]^{2} \\
= & \sum_{\substack{r_{1} r_{2} \in E\left(Q_{k}\left(G_{1}\right)\right) \\
r_{1} r_{2} \in V\left(Q_{k}\left(G_{1}\right)\right)-V\left(G_{1}\right)}} \sum_{s \in V\left(G_{2}\right)}\left[d\left(r_{1}\right)+d\left(r_{1}\right) d(s)+d\left(r_{2}\right)+d\left(r_{2}\right) d(s)\right]^{2} \\
= & \sum_{\substack{r_{1} r_{2} \in E\left(Q_{k}\left(G_{1}\right)\right) \\
r_{1}, r_{2} \in V\left(Q_{k}\left(G_{1}\right)\right)-\left(G_{1}\right)}} \sum_{s \in V\left(G_{2}\right)}\left[d\left(r_{1}\right)^{2}+d\left(r_{2}\right)^{2}+d(s)^{2}\left(d\left(r_{1}\right)^{2}+d\left(r_{2}\right)^{2}\right)+2 d(s)\left(d\left(r_{1}\right)^{2}+d\left(r_{2}\right)^{2}\right)\right] \\
& +2\left[\left(d\left(r_{1}\right)+d\left(r_{1}\right) d(s)\right)\left(d\left(r_{2}\right)+d\left(r_{2}\right) d(s)\right)\right], \\
D_{3}= & \sum_{\substack{r_{1} r_{2} \in E\left(Q\left(G_{1}\right)\right) \\
r_{1}, r_{2} \in V\left(Q\left(G_{1}\right)\right)-V\left(G_{1}\right)}}\left[d^{2}\left(r_{1}\right)+d^{2}\left(r_{2}\right)\right] .
\end{aligned}
$$

In $D_{3}$, coefficient of

$$
d^{2}(u)=2\left(\begin{array}{c}
2 \\
d_{G_{1}}(u)
\end{array}\right)+\sum_{v \in N(u)} d(v)-d(u)=d^{2}(u)-2 d(u)+\sum_{v \in N(u)} d(v) .
$$

Therefore,

$$
\sum_{u \in V\left(G_{1}\right)} d^{2}(u)=M_{4}\left(G_{1}\right)-2 F\left(G_{1}\right)+\sum_{u \in V\left(G_{1}\right)} d^{2}(u) \sum_{v \in N(u)} d(v) .
$$


For coefficient of $d(u) d(v)$, let $r_{1} r_{2} \epsilon E\left(Q\left(G_{1}\right)\right)$ with $r_{1}=$ $u v$ and $r_{2}=w z$. As $r_{1} r_{2} \in E\left(Q\left(G_{1}\right)\right)$, we have either $v=w$ or $z$ or $u=w$ or $z$. So, $u v$ is adjacent to all those vertices in $G_{1}$ which are adjacent to $u$ and $v$. Consequently, the number of such $d(u) d(v)$ is $(d(u)+d(v)-2)$. Therefore,

$$
\begin{aligned}
2 \sum_{u v \in E\left(G_{1}\right)} d(u) d(v) & =2 \sum_{u v \in E\left(G_{1}\right)}(d(u)+d(v)-2) \mathrm{d} u \mathrm{~d} v \\
& =2 \sum_{u v \in E\left(G_{1}\right)}(d(u)+d(v)) d(u) d(v)-4 \sum_{u v \in E\left(G_{1}\right)} d(u) d(v)=2 M_{2}\left(G_{1}\right)-4 M_{2}\left(G_{1}\right),
\end{aligned}
$$

so

$$
\begin{aligned}
D_{3}= & M_{4}\left(G_{1}\right)-2 F\left(G_{1}\right)+\sum_{u \in V\left(G_{1}\right)} d^{2}(u) \sum_{v \in N(u)} d(v)+2 M_{2}\left(G_{1}\right)-4 M_{2}\left(G_{1}\right), \\
\sum_{B_{4}}= & (k)\left[n_{G_{2}}+4 e_{G_{2}}+M_{1}\left(G_{2}\right)\right]\left[M_{4}\left(G_{1}\right)-2 F\left(G_{1}\right)+2 M_{2}\left(G_{1}\right)-4 M_{2}\left(G_{1}\right)+\sum_{u \in V\left(H_{1}\right)} d^{2}(u) \sum_{v \in N(u)} d(v)\right] \\
& +2\left[(k)\left[n_{G_{2}}+4 e_{G_{2}}+M_{1}\left(G_{2}\right)\right]\left[\frac{1}{2} \sum_{v \in V\left(G_{1}\right)}\left(d_{G_{1}}^{4}(v)-d_{G_{1}}^{3}(v)\right)+\sum_{u v \in V\left(G_{1}\right)} t d_{G_{1}}(u) d_{G_{1}}(v)\right]\right. \\
& \left.\left.+\sum_{v \in V\left(G_{1}\right)} d_{G_{1}}^{2}(v) \sum_{\substack{v \in V\left(G_{1}\right) \\
u v \in E\left(G_{1}\right)}} d_{G_{1}}(u)-2 M_{2}\left(G_{1}\right)\right]\right],
\end{aligned}
$$

where $t$ is the number of neighbors which are common vertices of $u$ and $v$ in $\left(G_{1}\right)$.

$$
\begin{aligned}
& \sum_{C}=\sum_{r_{1} r_{2} \in E\left(Q_{k}\left(G_{1}\right)\right)} \sum_{s_{1} s_{2} \in V\left(G_{2}\right)}\left[d\left(r_{1}, s_{1}\right)+d\left(r_{2}, s_{2}\right)\right]^{2}=\sum_{\substack{r_{1} r_{2} \in E\left(Q_{k}\left(G_{1}\right)\right) \\
r_{1} \in V\left(G_{1}\right), r_{2} \in V\left(Q_{k}\left(G_{1}\right)\right)-V\left(G_{1}\right)}} \sum_{s_{1} s_{2} \in V\left(G_{2}\right)}\left[d\left(r_{1}, s_{1}\right)+d\left(r_{2}, s_{2}\right)\right]^{2} \\
& +\sum_{r_{1} r_{2} \in E\left(Q_{k}\left(G_{1}\right)\right)} \sum_{s_{1} s_{2} \in V\left(G_{2}\right)}\left[d\left(r_{1}, s_{1}\right)+d\left(r_{2}, s_{2}\right)\right]^{2}=\sum_{C_{1}}+\sum_{C_{2}} \text {, } \\
& r_{1} \in V\left(G_{1}\right), r_{2} \in V\left(Q_{k}\left(G_{1}\right)\right)-V\left(G_{1}\right) \\
& \sum_{C_{1}}=\sum_{\substack{r_{1} r_{2} \in E\left(Q_{k}\left(G_{1}\right)\right) \\
r_{1} \in V\left(G_{1}\right), r_{2} \in V\left(Q_{k}\left(G_{1}\right)\right)-V\left(G_{1}\right)}} \sum_{s_{1} s_{2} \in V\left(G_{2}\right)}\left[d\left(r_{1}, s_{1}\right)+d\left(r_{2}, s_{2}\right)\right]^{2} \\
& =\sum_{\substack{r_{1} r_{2} \in E\left(Q_{k}\left(G_{1}\right)\right) \\
r_{1} \in V\left(G_{1}\right), r_{2} \in V\left(Q_{k}\left(G_{1}\right)\right)-V\left(G_{1}\right)}} \sum_{\substack{s_{1} s_{2} \in V\left(G_{2}\right)\\
}}\left[d\left(r_{1}\right)+d\left(s_{1}\right)+d\left(r_{1}\right) d\left(s_{1}\right)+d\left(r_{2}\right)+d\left(s_{2}\right)+d\left(r_{2} s_{2}\right)\right]^{2} \\
& =\sum_{\substack{r_{1} r_{2} \in E\left(Q_{k}\left(G_{1}\right)\right) \\
r_{1} \in V\left(G_{1}\right), r_{2} \in V\left(Q_{k}\left(G_{1}\right)\right)-V\left(G_{1}\right)}} \sum_{\substack{s_{1} s_{2} \in V\left(G_{2}\right)\\
}}\left[\left(d\left(r_{1}\right)+d\left(s_{1}\right)+d\left(r_{1}\right) d\left(s_{1}\right)\right)^{2}+\left(d\left(r_{2}\right)+d\left(r_{2}\right) d\left(s_{2}\right)\right)^{2}\right.
\end{aligned}
$$




$$
\begin{aligned}
& \left.+2\left[d\left(r_{1}, s_{1}\right) d\left(r_{2}, s_{2}\right)\right]\right] \\
= & 6\left[e_{G_{2}}+M_{1}\left(G_{2}\right)\right] F\left(G_{1}\right)+3 F\left(G_{1}\right) F\left(G_{2}\right)+2 M_{1}\left(G_{1}\right) M_{1}\left(G_{2}\right)+2\left[e_{G_{1}}+M_{1}\left(G_{1}\right)+2 M_{2}\left(G_{1}\right)\right] F\left(G_{2}\right)+8 M_{2}\left(G_{1}\right) \\
& \times\left[e_{G_{2}}+M_{1}\left(G_{2}\right)\right]+2\left[\left[M_{3}\left(G_{1}\right)+2 M_{2}\left(G_{1}\right)\right]\left[2 e_{G_{2}}+2 M_{1}\left(G_{2}\right)+2 M_{2}\left(G_{2}\right)\right]+2 M_{1}\left(G_{1}\right)\left[2 M_{2}\left(G_{2}\right)+M_{1}\left(G_{2}\right)\right]\right] .
\end{aligned}
$$

Now, assume $\Sigma_{C_{2}}=\Sigma_{C_{3}}+\Sigma_{C_{4}}$ as follows:

$$
\begin{aligned}
& \sum_{C_{3}}=\sum_{s_{1} s_{2} \in V\left(G_{2}\right)} \sum_{\substack{r_{1} r_{r_{2}} \in E\left(Q_{k}\left(G_{1}\right)\right) \\
r_{1} \in V\left(G_{1}\right), r_{2} \in V\left(Q_{k}\left(G_{1}\right)\right)-V\left(G_{1}\right)}}\left[d\left(r_{1}, s_{1}\right)+d\left(r_{2}, s_{2}\right)\right]^{2} \\
& =\sum_{s_{1} s_{2} \in V\left(G_{2}\right)} \sum_{\substack{r_{1} r_{2} \in E\left(Q_{k}\left(G_{1}\right)\right) \\
r_{1} \in V\left(G_{1}\right), r_{2} \in V\left(Q_{k}\left(G_{1}\right)\right)-V\left(G_{1}\right)}}\left[d\left(r_{1}\right)+d\left(r_{1}\right) d\left(s_{1}\right)+d\left(r_{2}\right)+d\left(r_{2}\right) d\left(s_{2}\right)\right]^{2} \\
& =\sum_{t_{1} t_{2} \in E\left(H_{2}\right)} \sum_{\substack{s_{1} s_{2} \in E\left(Q_{k}\left(H_{1}\right)\right) \\
s_{1}, s_{2} \in V\left(Q_{k}\left(H_{1}\right)-H_{1}\right)}}\left[\left(d\left(s_{1}\right)+d\left(s_{1}\right) d\left(t_{1}\right)\right)^{2}+\left(d\left(s_{2}\right)+d\left(s_{2}\right) d\left(t_{2}\right)\right)^{2}\right] \\
& +2\left[\left(d\left(r_{1}\right)+d\left(r_{1}\right) d(s)\right)\left(d\left(r_{2}\right)+d\left(r_{2}\right) d(s)\right)\right] \\
& =2(k-1)\left[\left(F\left(G_{1}\right)+2 M_{2}\left(G_{1}\right)\right)\right]\left[2 e_{G_{2}}+F\left(G_{2}\right)+2 M_{1}\left(G_{2}\right)\right]+2(k-1)\left[2 e_{G_{2}}+2 M_{1}\left(G_{2}\right)+2 M_{2}\left(G_{2}\right)\right]\left[M_{3}\left(G_{1}\right)+2 M_{2}\left(G_{1}\right)\right], \\
& \sum C_{4}=\sum_{s_{1} s_{2} \in V\left(G_{2}\right)} \sum_{\substack{r_{1} r_{2} \in E\left(Q_{k}\left(G_{1}\right)\right) \\
r_{1}, r_{2} \in V\left(Q_{k}\left(G_{1}\right)\right)-V\left(G_{1}\right)}}\left[d\left(r_{1}, s_{1}\right)+d\left(r_{2}, s_{2}\right)\right]^{2} \\
& =\sum_{s_{1} s_{2} \in V\left(G_{2}\right)} \sum_{\substack{r_{1} r_{2} \in E\left(Q_{k}\left(G_{1}\right)\right) \\
r_{1}, r_{2} E V\left(Q_{k}\left(G_{1}\right)\right)-V\left(G_{1}\right)}}\left[d\left(r_{1}\right)+d\left(r_{2}\right)+d\left(r_{1}\right) d\left(s_{1}\right)+d\left(r_{2}\right) d\left(s_{2}\right)\right]^{2} \\
& =\sum_{s_{1} s_{2} \in \mathscr{E}\left(G_{2}\right)} \sum_{\substack{r_{1} r_{2} \in E\left(Q_{k}\left(G_{1}\right)\right) \\
r_{1}, r_{2} \in V\left(Q_{k}\left(G_{1}\right)\right)-V\left(G_{1}\right)}}\left[\left(d\left(r_{1}\right)+d\left(r_{1}\right) d\left(s_{1}\right)\right)^{2}+\left(d\left(r_{2}\right)+d\left(r_{2}\right) d\left(s_{2}\right)\right)^{2}\right] \\
& +2\left[\left(d\left(r_{1}\right)+d\left(r_{1}\right) d(s)\right)\left(d\left(r_{2}\right)+d\left(r_{2}\right) d(s)\right)\right] \\
& =(k)\left[2 e_{G_{2}}+F\left(G_{2}\right)+2 M_{1}\left(G_{2}\right)\right]\left[M_{4}\left(G_{1}\right)-2 F\left(G_{1}\right)+2 M_{2}\left(G_{1}\right)-4 M_{2}\left(G_{1}\right)+\sum_{u \in V\left(G_{1}\right)} d^{2}(u) \sum_{v \in N(u)} d(v)\right] \\
& +(2 k)\left[2 e_{G_{2}}+2 M_{1}\left(G_{2}\right)+2 M_{2}\left(G_{2}\right)\right]\left[\frac{1}{2} \sum_{v \in V\left(G_{1}\right)}\left(d_{G_{1}}^{4}(v)-d_{G_{1}}^{3}(v)\right)+\sum_{u v \in V\left(G_{1}\right)} t d_{G_{1}}(u) d_{G_{1}}(v)\right. \\
& \left.+\sum_{u \in V\left(G_{1}\right)} d_{G_{1}}^{2}(v) \sum_{\substack{u \in V\left(G_{1}\right) \\
u v \in E\left(G_{1}\right)}} d_{G_{1}}(u)-2 M_{2}\left(G_{1}\right)\right]
\end{aligned}
$$


where $t$ is the number of neighbors which are common vertices of $u$ and $v$ in $\left(G_{1}\right)$.

Theorem 4. For $k \geq 1$, the HZ-index of $G_{1} \otimes_{T_{k}} G_{2}$ is

Thus, we arrive at our desired result.

$$
\begin{aligned}
\mathrm{HZ}\left(G_{1} \bigotimes_{T_{k}} G_{2}\right)= & 2(k-1)\left[F\left(G_{1}\right)+2 M_{2}\left(G_{1}\right)\right]\left[n_{G_{2}}+3 M_{1}\left(G_{2}\right)+6 e_{G_{2}}+F\left(G_{2}\right)\right]+k\left[n_{G_{2}}\right. \\
& \left.+6 e_{G_{2}}+3 M_{1}\left(G_{2}\right)+F\left(G_{2}\right)\right]\left[M_{4}\left(G_{1}\right)-2 F\left(G_{1}\right)+2 M_{2}\left(G_{1}\right)-4 M_{2}\left(G_{1}\right)\right. \\
& \left.+\sum_{u \in V\left(G_{1}\right)} d^{2}(u) \sum_{v \in N(u)} d v\right]+\left[F\left(G_{1}\right)+2 M_{2}\left(G_{1}\right)\right]\left[2 n_{G_{2}}+6 M_{1}\left(G_{2}\right)+12 e_{G_{2}}+2 F\left(G_{2}\right)\right] \\
& +4 F\left(G_{1}\right)\left[2 n_{G_{2}}+6 M_{1}\left(G_{2}\right)+12 e_{G_{2}}+2 F\left(G_{2}\right)\right]+F\left(G_{2}\right)\left[n_{G_{1}}+12 M_{1}\left(G_{1}\right)\right. \\
& \left.\left.+12 e_{G_{2}}\right]+12 e_{G_{1}} M_{1}\left(G_{2}\right)+16 e_{G_{2}} M_{1}\left(G_{1}\right)+20 M_{1}\left(G_{1}\right) M_{1}\left(G_{2}\right)\right] \\
& +2\left[k [ n _ { G _ { 2 } } + 6 e _ { G _ { 2 } } + 3 M _ { 1 } ( G _ { 2 } ) 2 M _ { 2 } ( G _ { 2 } ) ] \left[\frac{1}{2} \sum_{v \in V\left(G_{1}\right)}\left(d_{G_{1}}^{4}(v)-d_{G_{1}}^{3}(v)\right)+\sum_{u v e V\left(G_{1}\right)} d_{G_{1}}(u) d_{G_{1}}(v)\right.\right. \\
& \left.+\sum_{v \in V\left(G_{1}\right)} d_{G_{1}}^{2}(v) \sum_{u \in V\left(G_{1}\right)} d_{G_{1}}(u)-2 M_{2}\left(G_{1}\right)\right]+M_{2}\left(G_{1}\right)\left[4 n_{G_{2}}+24 e_{G_{2}}+8 M_{2}\left(G_{2}\right)+12 M_{1}\left(G_{2}\right)\right] \\
& +k\left[M_{3}\left(G_{1}\right)+2 M_{2}\left(G_{1}\right)\right]\left[6 e_{G_{2}}+n_{G_{2}}+3 M_{1}\left(G_{2}\right)+2 M_{2}\left(G_{2}\right)\right]+5 M_{1}\left(G_{2}\right) e_{G_{1}} \\
& \left.+M_{2}\left(G_{2}\right)\left[10 e_{G_{1}}+n_{G_{1}}\right]+M_{1}\left(G_{1}\right)\left[10 e_{G_{2}}+11 M_{1}\left(G_{2}\right)+10 M_{2}\left(G_{2}\right)\right]\right] .
\end{aligned}
$$

\section{Applications and Discussion}

(i) $S_{1}$-sum:

Using $k=1$, in Theorems $1-4$, the results are obtained for the generalized $D_{1}$-sum graphs as follows:

$$
\begin{aligned}
\mathrm{HZ}\left(G_{1} \unrhd_{S_{1}} G_{2}\right)= & {\left[n_{G_{2}}+3 M_{1}\left(G_{2}\right)+6 e_{G_{2}}\right] F\left(G_{1}\right)+\left[n_{G_{1}}+3 M_{1}\left(G_{1}\right)+14 e_{G_{1}}\right] F\left(G_{2}\right)+6 e_{G_{2}} M_{1}\left(G_{1}\right)+30 e_{G_{1}} M_{1}\left(G_{2}\right) } \\
& +6 M_{1}\left(G_{1}\right) M_{1}\left(G_{2}\right)+48 e_{G_{1}} e_{G_{2}}+F\left(G_{1}\right) F\left(G_{2}\right)+8 n_{G_{2}} e_{G_{1}}+2\left[[ M _ { 2 } ( G _ { 1 } ) + 4 e _ { G _ { 1 } } ] \left[5 e_{G_{2}}+3 M_{1}\left(G_{2}\right)\right.\right. \\
& \left.\left.+2 M_{2}\left(G_{2}\right)+n_{G_{2}}\right]+14 e_{G_{1}} M_{1}\left(G_{2}\right)+M_{2}\left(G_{2}\right)\right]\left[12 e_{G_{1}}+n_{G_{1}}\right]+M_{1}\left(G_{1}\right)\left[e_{G_{2}}+M_{1}\left(G_{2}\right)+M_{2}\left(G_{2}\right)\right] \\
& \left.+8 e_{G_{1}} e_{G_{2}}\right] .
\end{aligned}
$$


TABLe 1: Hyper-Zagreb index of $F_{1}$-sum path graphs.

\begin{tabular}{lcccc}
\hline$\left[n_{1}, n_{2}\right]$ & $\mathrm{HZ}\left(P_{n_{1}} \otimes_{S_{1}} P_{n_{2}}\right)$ & $\mathrm{HZ}\left(P_{n_{1}} \otimes_{R_{1}} P_{n_{2}}\right)$ & $\mathrm{HZ}\left(P_{n_{1}} \otimes_{\mathrm{Q}_{1}} P_{n_{2}}\right)$ & $\mathrm{HZ}\left(P_{n_{1}} \otimes_{T_{1}} P_{n_{2}}\right)$ \\
\hline$(3,3)$ & 4144 & 11870 & 8834 & 16168 \\
$(4,4)$ & 11040 & 30260 & 24500 & 47180 \\
$(5,5)$ & 21232 & 62122 & 46516 & 89230 \\
$(6,6)$ & 33696 & 99344 & 75416 & 145204 \\
$(7,7)$ & 51384 & 147638 & 110384 & 212318 \\
\hline
\end{tabular}

(ii) $R_{1}$-sum:

$$
\begin{aligned}
\operatorname{HZ}\left(G_{1} \unrhd_{R_{1}} G_{2}\right)= & 8\left[n_{G_{2}}+6 e_{G_{2}}\right] F\left(G_{1}\right)+\left[n_{G_{1}}+20 e_{G_{1}}\right] F\left(G_{2}\right)+8 F\left(G_{1}\right) F\left(G_{2}\right)+24 e_{G_{2}} M_{1}\left(G_{1}\right)+36 e_{G_{1}} M_{1}\left(G_{2}\right) \\
& +24 M_{1}\left(G_{1}\right) M_{1}\left(G_{2}\right)+24 F\left(G_{1}\right) M_{1}\left(G_{2}\right)+8 n_{G_{2}} e_{G_{1}}+48 e_{G_{1}} e_{G_{2}}+12 F\left(G_{2}\right) M_{1}\left(G_{1}\right) \\
& +2\left[2 M_{1}\left(G_{1}\right)\left[4 e_{G_{2}}+6 M_{1}\left(G_{2}\right)+2 M_{2}\left(G_{2}\right)\right]+4 e_{G_{1}}\left[3 M_{1}\left(G_{2}\right)+3 M_{2}\left(G_{2}\right)+4 e_{G_{2}}\right]\right. \\
& +\left[M_{2}\left(R_{1}\left(G_{1}\right)\right)-4 M_{2} G_{1}\right]\left[6 e_{G_{2}}+3 M_{1}\left(G_{2}\right)+2 M_{2}\left(G_{2}\right)+n_{G_{2}}\right]+e_{G_{1}} M_{1}\left(G_{2}\right) \\
& \left.+4 M_{2}\left(G_{1}\right)\left[n_{G_{2}}+6 e_{G_{2}}+3 M_{1}\left(G_{2}\right)\right]+M_{2}\left(G_{2}\right)\left[n_{G_{1}}+2 e_{G_{1}}\right]\right] .
\end{aligned}
$$

(iii) $Q_{1}$-sum:

$$
\begin{aligned}
\mathrm{HZ}\left(G_{1} \unrhd_{Q_{1}} G_{2}\right)= & 2\left[[ n _ { G _ { 2 } } + 6 e _ { G _ { 2 } } + 3 M _ { 1 } ( G _ { 2 } ) + 2 M _ { 2 } ( G _ { 2 } ) ] \left[\frac{1}{2} \sum_{v \in V\left(G_{1}\right)}\left(d_{G_{1}}^{4}(v)-d_{G_{1}}^{3}(v)\right)+\sum_{u v \in V\left(G_{1}\right)} t d_{G_{1}}(u) d_{G_{1}}(v)\right.\right. \\
& \left.+\sum_{v \in V\left(G_{1}\right)} d_{G_{1}}^{2}(v) \sum_{u \in V\left(G_{1}\right)} d_{G_{1}}(u)-2 M_{2}\left(G_{1}\right)\right]+M_{2}\left(G_{2}\right)\left[4 e_{G_{1}}+n_{G_{1}}\right]+2 e_{G_{1}} M_{1}\left(G_{2}\right)+M_{1}\left(G_{1}\right) \\
& \left.\times\left[5 e_{2}+5 M_{1}\left(G_{2}\right)+5 M_{2}\left(G_{2}\right)\right]+\left[M_{3}\left(G_{1}\right)+2 M_{2}\left(G_{1}\right)\right]\left[6 e_{G_{2}}+3 M_{1}\left(G_{2}\right)+2 M_{2}\left(G_{2}\right)+n_{G_{2}}\right]\right] \\
& +\left[n_{G_{2}}+e_{G_{2}}+3 M_{1}\left(G_{2}\right)+F\left(G_{2}\right)\right]\left[M_{4}\left(G_{1}\right)-2 F\left(G_{1}\right)+2 M_{2}\left(G_{1}\right)-4 M_{2}\left(G_{1}\right)+\sum_{u \in V\left(G_{1}\right)} d^{2}(u)\right. \\
& \left.\times \sum_{v \in N(u)} d(v)\right]+6 e_{G_{2}} M_{1}\left(G_{1}\right)+10 e_{G_{2}} F\left(G_{2}\right)+3 F\left(G_{1}\right) F\left(G_{2}\right)+6 M_{1}\left(G_{1}\right) M_{1}\left(G_{2}\right)+F\left(G_{2}\right)\left[n_{G_{1}}\right. \\
& \left.+3 M_{1}\left(G_{1}\right)+6 e_{G_{2}}+4 M_{2}\left(G_{1}\right)\right]+F\left(G_{1}\right)\left[n_{G_{2}}+7 M_{1}\left(G_{2}\right)\right]+6 e_{G_{2}} M_{2}\left(G_{1}\right)+8 M_{2}\left(G_{1}\right)\left[e_{G_{2}}+M_{1}\left(G_{2}\right)\right] .
\end{aligned}
$$




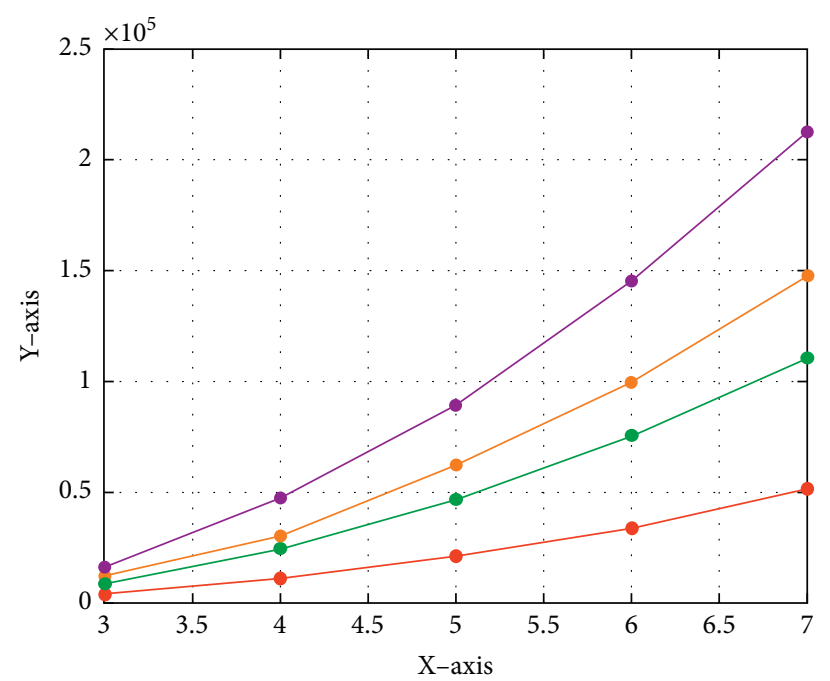

FIGURE 4: Graphical representation of $\operatorname{HZ}\left(P_{n_{1}} \rrbracket_{S_{1}} P_{m}\right), \mathrm{HZ}\left(P_{n_{1}} \rrbracket_{R_{1}} P_{m}\right), \mathrm{HZ}\left(P_{n_{1}} \rrbracket_{Q_{1}} P_{m}\right)$, and $\operatorname{HZ}\left(P_{n_{1}} \rrbracket_{T_{1}} P_{m}\right)$ in red, green, orange, and purple colour, respectively.

(iv) $T_{1}$-sum:

$$
\begin{aligned}
\mathrm{HZ}\left(G_{1} \bigotimes_{T_{1}} G_{2}\right)= & {\left[n_{G_{2}}+6 e_{G_{2}}+3 M_{1}\left(G_{2}\right)+F\left(G_{2}\right)\right]\left[M_{4}\left(G_{1}\right)-2 F\left(G_{1}\right)+2 M_{2}\left(G_{1}\right)-4 M_{2}\left(G_{1}\right)+\sum_{u \in V\left(G_{1}\right)} d^{2}(u)\right.} \\
& \left.\times \sum_{v \in N(u)} d(v)\right]+\left[F\left(G_{1}\right)+2 M_{2}\left(G_{1}\right)\right]\left[2 n\left(G_{2}\right)+6 M_{1}\left(G_{2}\right)+12 e_{G_{2}}+2 F\left(G_{2}\right)\right]+4 F\left(G_{1}\right)\left[2 n_{G_{2}}\right. \\
& \left.+6 M_{1}\left(G_{2}\right)+12 e_{G_{2}}+2 F\left(G_{2}\right)\right]+F\left(G_{2}\right)\left[n_{G_{1}}+12 M_{1}\left(G_{1}\right)+12 e_{G_{2}}\right]+12 e_{G_{1}} M_{1}\left(G_{2}\right)+16 e_{G_{2}} M_{1}\left(G_{1}\right) \\
& +20 M_{1}\left(G_{1}\right) M_{1}\left(G_{2}\right)+2\left[[ n _ { G _ { 2 } } + 6 e _ { G _ { 2 } } + 3 M _ { 1 } ( G _ { 2 } ) + 2 M _ { 2 } ( G _ { 2 } ) ] \left[\frac{1}{2} \sum_{v e V\left(G_{1}\right)}\left(d_{G_{1}}^{4}(v)-d_{G_{1}}^{3}(v)\right)\right.\right. \\
& \left.+\sum_{u v \in V\left(G_{1}\right)} t d_{G_{1}}(u) d_{G_{1}}(v)+\sum_{v \in V\left(G_{1}\right)} d_{G_{1}}^{2}(v) \sum_{u \in V\left(G_{1}\right)} d_{G_{1}}(u)-2 M_{2}\left(G_{1}\right)\right]+\left[M_{3}\left(G_{1}\right)+2 M_{2}\left(G_{1}\right)\right] \\
& +\left[6 e_{G_{2}}+n_{G_{2}}+3 M_{1}\left(G_{2}\right)+2 M_{2}\left(G_{2}\right)\right]+5 M_{1}\left(G_{2}\right) e_{G_{1}}+M_{1}\left(G_{1}\right)\left[10 e_{G_{2}}+11 M_{1}\left(G_{2}\right)+10 M_{2}\left(G_{2}\right)\right] \\
& \left.+M_{2}\left(G_{2}\right)\left[10 e_{G_{1}}+n_{G_{1}}\right]+M_{2}\left(G_{1}\right)\left[4 n_{G_{2}}+24 e_{G_{2}}+8 M_{2}\left(G_{2}\right)+12 M_{1}\left(G_{2}\right)\right]\right] .
\end{aligned}
$$


Now, we present tabular form in Table 1 and graphical representation in Figure 4 of path graphs for $k=1$.

Finally, we close this section with the comment that the problem is still open for other topological indices and product of graphs, in particular the general randic index of $F_{k}$-sum graphs under corona product.

\section{Data Availability}

The data are included within this paper and are available from the corresponding author upon request.

\section{Conflicts of Interest}

The authors declare that they have no conflicts of interest.

\section{Acknowledgments}

The authors extend their appreciation to the Deanship of Scientific Research at Jouf University for funding this work through research grant no. DSR-2021-03-0222.

\section{References}

[1] I. Gutman and O. Polansky, Mathematical Concepts in Organic Chemistry, Springer-Verlag, Berlin, Germany, 1986.

[2] R. Todeschini, V. Consonni, R. Mannhold, H. Kubinyi, and H. Timmerman, Handbook of Molecular Descriptors, WileyVCH, Weinheim, Germany, 2002.

[3] D. M. Cvetkocic, M. Doob, and H. Sachs, Spectra of Graphs: Theory and Application, Academic Press, New York, NY, USA, 1980.

[4] G. Rücker and C. Rucker, "On topological indices, boiling points, and cycloalkanes," Journal of Chemical Information and Computer Sciences, vol. 39, no. 5, pp. 788-802, 1999.

[5] M. Randic, "Characterization of molecular branching," Journal of the American Chemical Society, vol. 97, no. 23, pp. 6609-6615, 1975.

[6] A. R. Matamala and E. Estrada, "Generalised topological indices: optimisation methodology and physico-chemical interpretation," Chemical Physics Letters, vol. 410, no. 4-6, pp. 343-347, 2005.

[7] F. Yan, Q. Shang, S. Xia, Q. Wang, and P. Ma, "Application of topological index in predicting ionic liquids densities by the quantitative structure property relationship method," Journal of Chemical \& Engineering Data, vol. 60, no. 3, pp. 734-739, 2015.

[8] L. H. Hall and L. B. Kier, Molecular Connectivity in Chemistry and Drug Research, Academic Press, Boston, MA, USA, 1976.

[9] H. Gonzalez-Diaz, S. Vilar, L. Santana, and E. Uriarte, "Medicinal chemistry and bioinformatics-current trends in drugs discovery with networks topological indices," Current Topics in Medicinal Chemistry, vol. 7, no. 10, pp. 1015-1029, 2007.

[10] M. V. Diudea, QSPR/QSAR Studies by Molecular Descriptors, NOVA, New York, NY, USA, 2001.

[11] J. Devillers and A. T. Balaban, Topological Indices and Related Descriptors in QSAR and QSPR, Gordon Breach, Amsterdam, Netherlands, 1999.

[12] H. Wiener, "Structural determination of paraffin boiling points," Journal of the American Chemical Society, vol. 69, no. 1, pp. 17-20, 1947.
[13] I. Gutman and N. Trinajstić, "Graph theory and molecular orbitals. Total $\varphi$-electron energy of alternant hydrocarbons," Chemical Physics Letters, vol. 17, no. 4, pp. 535-538, 1972.

[14] I. Gutman, "Degree-based topological indices," Croatica Chemica Acta, vol. 86, no. 4, pp. 351-361, 2013.

[15] Z. Ahmad, M. Naseem, M. Naseem, M. K. Jamil, M. F. Nadeem, and S. Wang, "Eccentric connectivity indices of titania nanotubes $\mathrm{TiO}_{2}$ [m;n]," Eurasian Chemical Communications, vol. 2, no. 6, pp. 712-721, 2020.

[16] S. Imran, M. Siddiqui, M. Imran, and M. Nadeem, "Computing topological indices and polynomials for line graphs," Mathematics, vol. 6, no. 8, p. 137, 2018.

[17] A. Aslam, Y. Bashir, S. Ahmad, and W. Gao, "On topological indices of certain dendrimer structures," Zeitschrift für Naturforschung A, vol. 72, no. 6, pp. 559-566, 2017.

[18] A. Ahmad, R. Hasni, K. Elahi, and M. A. Asim, "Polynomials of degree-based indices for swapped networks modeled by optical transpose interconnection system," IEEE Access, vol. 8, pp. 214293-214299, 2020.

[19] A. Ahmad, "Comparative study of ve-degree and ev-degree topological descriptors for benzene ring embedded in p-typesurface in 2D network," Polycyclic Aromatic Compounds, pp. 1-10, 2020.

[20] H. M. A. Siddiqui, "Computation of Zagreb indices and Zagreb polynomials of sierpinski graphs," Hacettepe Journal of Mathematics and Statistics, vol. 49, no. 2, pp. 754-765, 2020.

[21] B. Zhou and N. Trinajstić, "On general sum-connectivity index," Journal of Mathematical Chemistry, vol. 47, no. 1, pp. $210-218,2010$.

[22] G. H. Shirdel, H. Rezapour, and A. M. Sayadi, "Hyper-Zagreb index of graph operations," Iranian Journal of Mathematical Chemistry, vol. 4, pp. 213-220, 2013.

[23] V. Anandkumar and R. R. Iyer, "On the hyper-Zagreb index of some operations on graphs," Indian Journal of Pure and Applied Mathematics, vol. 112, pp. 239-252, 2017.

[24] B. Basavanagoud and S. Patil, "A note on hyper-Zagreb index of graph operations," Journal of Applied Mathematical Chemistry, vol. 7, pp. 89-92, 2016.

[25] W. Gao, M. K. Jamil, and M. R. Farahani, “The Hyper-Zagreb index and some graph operations," Journal of Applied Mathematics and Computer Science, vol. 54, pp. 263-275, 2016.

[26] W. Yan, B.-Y. Yang, and Y.-N. Yeh, “The behavior of Wiener indices and polynomials of graphs under five graph decorations," Applied Mathematics Letters, vol. 20, no. 3, pp. 290-295, 2007.

[27] M. Eliasi and B. Taeri, "Four new sums of graphs and their Wiener indices," Discrete Applied Mathematics, vol. 157, no. 4, pp. 794-803, 2009.

[28] H. Deng, D. Sarala, S. K. Ayyaswamy, and S. Balachandran, "The Zagreb indices of four operations on graphs," Applied Mathematics and Computation, vol. 275, pp. 422-431, 2016.

[29] S. Akhter and M. Imran, "Computing the forgotten topological index of four operations on graphs," AKCE International Journal of Graphs and Combinatorics, vol. 14, no. 1, pp. 70-79, 2017.

[30] Y.-M. Chu, S. Javed, M. Javaid, and M. Kamran Siddiqui, “On bounds for topological descriptors of $\varphi$-sum graphs," Journal of Taibah University for Science, vol. 14, no. 1, pp. 1288-1301, 2020.

[31] J.-B. Liu, S. Javed, M. Javaid, and K. Shabbir, "Computing first general Zagreb index of operations on graphs," IEEE Access, vol. 7, pp. 47494-47502, 2019. 
[32] J.-B. Liu, M. Javaid, and H. M. Awais, "Computing Zagreb indices of the subdivision-related generalized operations of graphs," IEEE Access, vol. 7, pp. 105479-105488, 2019.

[33] M. Javaid, S. Javed, A. M. Alanazi, and M. R. Alotaibi, "Computing analysis of Zagreb indices for generalized sum graphs under strong product," Journal of Chemistry, vol. 2021, Article ID 6663624, 20 pages, 2021.

[34] B. Furtula and I. Gutman, "A forgotten topological index," Journal of Mathematical Chemistry, vol. 53, no. 4, pp. 11841190, 2015.

[35] M. Alsharafi, M. Shubatah, and A. Alameri, "The hyperZagreb index of some complement graphs," Advances in Mathematics: Scientific Journal, vol. 9, no. 6, pp. 3631-3642, 2020.

[36] I. Gutman, B. Ruscic, N. Trinajstic, and C. F. Wilcox, "Graph theory and molecular orbitals. XII. Acyclic polyenes," The Journal of Chemical Physics, vol. 62, no. 9, pp. 3399-3405, 1975.

[37] I. Gutman and N. Trinajstic, "Graph theory and molecular orbitals," in New Concept, vol. 2, pp. 49-93, Springer, Berlin, Heidelberg, 1973. 